MATHEMATICS OF COMPUTATION

Volume 74, Number 249, Pages 25-52

S 0025-5718(04)01662-X

Article electronically published on April 28, 2004

\title{
A FINITE ELEMENT METHOD FOR NEARLY INCOMPRESSIBLE ELASTICITY PROBLEMS
}

\author{
DIETRICH BRAESS AND PINGBING MING
}

\begin{abstract}
A finite element method is considered for dealing with nearly incompressible material. In the case of large deformations the nonlinear character of the volumetric contribution has to be taken into account. The proposed mixed method avoids volumetric locking also in this case and is robust for $\lambda \rightarrow \infty$ (with $\lambda$ being the well-known Lamé constant). Error estimates for the $L^{\infty}$-norm are crucial in the control of the nonlinear terms.
\end{abstract}

\section{INTRODUCTION}

Isoparametric low-order elements are very popular in solid mechanics due to their simplicity. A major disadvantage, however, is the so-called locking effect [2]. Specifically volume locking is encountered when the material is nearly incompressible. In case of linear elasticity it is now well known how to overcome locking numerically, and several popular methods have been shown to be equivalent [6, 30]. Recently, the mathematical treatment has also been simplified [8].

The situation is less satisfactory in nonlinear elasticity. In the present paper we shall consider a nearly incompressible neo-Hookean material [27, 37]. Let $\mathbf{v}$ be a displacement field in a bounded domain $\Omega \subset \mathbb{R}^{2}$ with smooth boundary. The stored energy and the load yield the total energy

(1.1) $\mathcal{J}_{\lambda}(\mathbf{v}):=\frac{C_{0}}{2} \int_{\Omega}\left(|\mathrm{Id}+\nabla \mathbf{v}|^{2}-2\right) d \mathbf{x}+\frac{\lambda}{2} \int_{\Omega}|\operatorname{det}(\operatorname{Id}+\nabla \mathbf{v})-1|^{2} d \mathbf{x}-\int_{\Omega} \mathbf{f} \mathbf{v} d \mathbf{x}$.

Here $C_{0}$ is a positive physical parameter related to the Lamé parameter $\mu$ while $\lambda$ is characteristic for the compressibility. In particular, here we have $\lambda \gg C_{0}$. For brevity, we let $C_{0} \equiv 1$ hereafter. This model (and its analysis) have a characteristic feature of nonlinear theories. We find the determinant $\operatorname{det}(\operatorname{Id}+\boldsymbol{\nabla} \mathbf{v})$ in expressions at those places where $\operatorname{div} v$ is encountered in linear theories. The resulting displacement $\mathbf{u}=\left(u_{1}, u_{2}\right)$ is characterized by the minimization of $\mathcal{J}_{\lambda}(\mathbf{v})$, i.e.,

$$
\mathcal{J}_{\lambda}(\mathbf{u})=\min _{\mathbf{v} \in \mathbf{W}} \mathcal{J}_{\lambda}(\mathbf{v})
$$

where $\mathbf{W}:=\left\{\mathbf{v} \in \mathbf{W}_{0}^{1,4}(\Omega) \mid \operatorname{det}(\operatorname{Id}+\nabla \mathbf{v})>0\right.$ a.e. in $\left.\Omega\right\}$.

The above minimization problem is frequently used in industry [23, 29] to model neo-Hookean nearly incompressible material. Natural rubber is a typical example for nearly incompressible material, and materials that undergo plastic deformations

Received by the editor November 7, 2001 and, in revised form, July 11, 2003.

2000 Mathematics Subject Classification. Primary 65N30, 74S05, 74B20.

Key words and phrases. Incompressible elasticity, Green's functions, $L^{\infty}$-estimates.

(C)2004 American Mathematical Society 
may also be considered as nearly incompressible. This model is also encountered when the hard incompressible constraint

$$
\operatorname{det}(\mathrm{Id}+\boldsymbol{\nabla} \mathbf{v})=1
$$

is tackled by a penalty term in a way introduced by Ogden [26]. The above constraint reduces to the well-known linear incompressible condition in linear elasticity or incompressible fluid dynamics, i.e.,

$$
\operatorname{div} \mathbf{v}=0 .
$$

The model may also be viewed as the 2D case of the Mooney-Rivlin material; we refer to [12] for examples of other materials. Proceeding along the same line as [22, Theorem II.2] (see also [32]), one can see that $\mathbf{u}$ and $p$ converge to the solutions for incompressible neo-Hookean material as $\lambda$ approaches $\infty$ if the body force $\mathbf{f} \in \mathbf{L}^{2}(\Omega)$.

An advantage of this model is that it is based upon a displacement-oriented formulation which facilitates the implementation. The model avoids hard constraints. On the other hand, there is a large parameter and the danger of locking. In order to avoid locking in the given nonlinear problem, we will employ a reduced energy functional when the discretization with finite elements of low order is performed. Let $\mathbf{X}_{h}$ be the finite elements space. The solution $\mathbf{u}_{h}$ will minimize the reduced energy functional

$$
\begin{aligned}
\mathcal{J}_{\lambda, h}(\mathbf{v}): & =\frac{1}{2} \int_{\Omega}\left(|\mathrm{Id}+\nabla \mathbf{v}|^{2}-2\right) d \mathbf{x}+\frac{\lambda}{2} \int_{\Omega}\left|\Pi_{0}(\operatorname{det}(\operatorname{Id}+\nabla \mathbf{v})-1)\right|^{2} d \mathbf{x} \\
& -\int_{\Omega} \mathbf{f} \mathbf{v} d \mathbf{x},
\end{aligned}
$$

where $\Pi_{0}$ denotes the $L^{2}$-projection onto some finite element space $M_{h}$.

The analysis will be based on an equivalent mixed method. To this end the pressure variable

$$
p:=\lambda(\operatorname{det}(\operatorname{Id}+\nabla \mathbf{u})-1)
$$

is introduced, and a saddle point formulation with a penalty term arises. While such a $u-p$ formulation is equivalent to the method with enhanced assumed strains in the linear case [6, 35, there are some differences in the nonlinear case. Instead of using a projection of the determinant of Id $+\nabla \mathbf{u}$, one may for instance consider projections of the four matrix elements. This will be exploited in a forthcoming paper.

The nonlinear problem will be dealt with in a homotopy argument. Since the nonlinearities are bounded by $L^{\infty}$-norms, the $L^{\infty}$-error has to be controlled for the homotopy. As a consequence, we obtain logarithmic terms with a higher power than in the linear case.

We emphasize that we do not require smallness of the nonlinearities. It will be enough to have a good discretization such that the nonlinear terms with the differences $\mathbf{u}-\mathbf{u}_{h}$ and $p-p_{h}$ can be controlled.

The paper is organized as follows. Some notations are introduced, the main result is stated, and the concept for its proof is outlined in Section 2, In Section 3 we provide some a priori estimates as well as the regularity estimates for the linearized problem. A special Clément interpolant is constructed in Section 4. The regularized Green's functions and their application to the nonlinear problem are 
detailed in Section [5] Section [6 establishes the final error bounds. Appendix A tackles the finite element approximation of the Green's functions.

In this paper, we will concentrate on a variant of the $4-1$ element [36]. The commonly used 9-3 element also fits into our analysis; we will comment on this element in Remarks 2.8 and 4.2 .

Throughout this paper, $C$ denotes a generic constant that is independent of $h$ and $\lambda$.

\section{Notations AND PRELIMinaries}

2.1. Problem setting. Let $W^{m, p}(\Omega)$ denote the standard Sobolev space of functions on $\Omega$ (see [20]) equipped with the norm and semi-norm

$$
\|v\|_{m, p}^{p}:=\sum_{k=0}^{m}|v|_{k, p}^{p} \quad \text { and } \quad|v|_{k, p}^{p}:=\int_{\Omega} \sum_{|\boldsymbol{\alpha}|=k}\left|\mathcal{D}^{\boldsymbol{\alpha}} v\right|^{p} d \mathbf{x} .
$$

Here $\boldsymbol{\alpha}=\left(\alpha_{1}, \alpha_{2}\right)$ is a multi-index whose components $\alpha_{i}$ are nonnegative integers, $|\boldsymbol{\alpha}|=\alpha_{1}+\alpha_{2}$, and $\mathcal{D}^{\boldsymbol{\alpha}}=\partial^{|\boldsymbol{\alpha}|} / \partial x_{1}^{\alpha_{1}} x_{2}^{\alpha_{2}}$. For brevity, if $p=2$, the subscript $p$ will be dropped and $W^{m, 2}(\Omega)$ is denoted as $H^{m}(\Omega)$. As usual, $H_{0}^{1}(\Omega)$ is the subspace of $H^{1}(\Omega)$ functions with vanishing traces on $\Gamma:=\partial \Omega$, and $\mathbf{H}_{0}^{1}(\Omega)$ is the corresponding space of 2 -vector-valued functions. More generally, we use boldface letters to denote the vector-valued spaces and operators. Moreover $H^{-1}(\Omega)$ is the dual space of $H_{0}^{1}(\Omega)$, and $\langle\cdot, \cdot\rangle$ denotes the dual pairing of $H_{0}^{1}(\Omega)$ and $H^{-1}(\Omega)$ or of $\mathbf{H}_{0}^{1}(\Omega)$ and $\mathbf{H}^{-1}(\Omega)$, respectively. The inner product in $L^{2}(\Omega)$ is $(\cdot, \cdot)_{\Omega}$ or for short $(\cdot, \cdot)$.

We set $\mathbf{X}:=\mathbf{H}_{0}^{1}(\Omega)$ and $M:=L^{2}(\Omega)$. As usual $\mathbf{X}^{\prime}$ and $M^{\prime}$ are the dual spaces.

For a vector $\mathbf{x} \in \mathbb{R}^{2}$, we set $\mathbf{x}^{\perp}:=\left(x_{2},-x_{1}\right)$. For vectors $\mathbf{x}=\left(x_{1}, x_{2}\right)$ and $\mathbf{y}=\left(y_{1}, y_{2}\right) \in \mathbb{R}^{2}, \mathbf{x} \otimes \mathbf{y}$ is a $2 \times 2$ matrix with elements $(\mathbf{x} \otimes \mathbf{y})_{i j}:=x_{i} y_{j}$. For a matrix $\mathbf{A}$, adj $\mathbf{A}:=(\operatorname{Cof} \mathbf{A})^{T}=\operatorname{Cof} \mathbf{A}^{T}$, where Cof $\mathbf{A}$ is the cofactor matrix of $\mathbf{A}$. A matrix product is defined by $\mathbf{A}: \mathbf{B}=\operatorname{tr}\left(\mathbf{A}^{T} \mathbf{B}\right)$. Note that

$$
\operatorname{det}(\mathbf{A}+\mathbf{B})=\operatorname{det} \mathbf{A}+\operatorname{Cof} \mathbf{A}: \mathbf{B}+\operatorname{det} \mathbf{B}
$$

holds for any $2 \times 2$ matrices.

Problem (1.2) still has an undesirable constraint. We consider the minimization of the elastic energy (1.2) over $\mathbf{W}_{0}^{1,4}(\Omega)$ instead of $\mathbf{W}$ and the restriction $\operatorname{det}(\operatorname{Id}+\boldsymbol{\nabla v})>0$ has to be checked a posteriori. Since zero boundary conditions are assumed, $\int_{\Omega}\left(|\mathrm{Id}+\boldsymbol{\nabla} \mathbf{v}|^{2}-2\right) d \mathbf{x}=\int_{\Omega}(\nabla \mathbf{v})^{2} d \mathbf{x}$. The Euler-Lagrange equation for the minimization of (1.1) is

$$
A(\mathbf{u}, \mathbf{v})=\langle\mathbf{f}, \mathbf{v}\rangle, \quad \forall \mathbf{v} \in \mathbf{W}_{0}^{1,4}(\Omega),
$$

with the nonlinear functional

$$
A(\mathbf{u}, \mathbf{v}):=(\boldsymbol{\nabla} \mathbf{u}, \boldsymbol{\nabla} \mathbf{v})+\lambda(\operatorname{det}(\mathrm{Id}+\boldsymbol{\nabla} \mathbf{u})-1, \operatorname{Cof}(\mathrm{Id}+\boldsymbol{\nabla} \mathbf{u}): \nabla \mathbf{v}) .
$$

For problems with more general boundary conditions, see [33].

By virtue of the Piola identity [12, Theorem 1.7.1],

$$
\operatorname{div} \operatorname{Cof} \mathbf{A}=\mathbf{0} \text {, }
$$

whenever $\mathbf{A}$ is the gradient of a smooth vector field. Therefore $A(\mathbf{u}, \mathbf{v})$ can be rewritten

$$
A(\mathbf{u}, \mathbf{v})=(\boldsymbol{\nabla} \mathbf{u}, \boldsymbol{\nabla} \mathbf{v})+\lambda(\operatorname{det}(\operatorname{Id}+\boldsymbol{\nabla} \mathbf{u})-1, \operatorname{div}(\operatorname{adj}(\operatorname{Id}+\boldsymbol{\nabla u}) \mathbf{v})) .
$$


We introduce $p:=\lambda(\operatorname{det}(\operatorname{Id}+\boldsymbol{\nabla u})-1)$ to put the minimizing problem (1.2) and the weak equations (2.2) into a saddle point formulation.

Problem 2.1. Find $(\mathbf{u}, p) \in \mathbf{W}_{0}^{1,4}(\Omega) \times M$ such that

$$
\begin{aligned}
a((\mathbf{u}, p), \mathbf{v}) & =\langle\mathbf{f}, \mathbf{v}\rangle, & & \forall \mathbf{v} \in \mathbf{W}_{0}^{1,4}(\Omega), \\
b(\mathbf{u}, q)-\lambda^{-1}(p, q) & =0, & & \forall q \in M,
\end{aligned}
$$

with

$$
\begin{aligned}
a((\mathbf{u}, p), \mathbf{v}) & :=(\boldsymbol{\nabla} \mathbf{u}, \boldsymbol{\nabla} \mathbf{v})+(p, \operatorname{div}(\operatorname{adj}(\operatorname{Id}+\boldsymbol{\nabla} \mathbf{u}) \mathbf{v})) \\
b(\mathbf{u}, q) & :=(q, \operatorname{det}(\operatorname{Id}+\boldsymbol{\nabla} \mathbf{u})-1) .
\end{aligned}
$$

As above, from the Piola identity we have $\operatorname{div}(\operatorname{Cof}(\operatorname{Id}+\nabla \mathbf{u}))=0$ and

$$
(p, \operatorname{div}(\operatorname{adj}(\operatorname{Id}+\boldsymbol{\nabla u}) \mathbf{v}))=(p, \operatorname{Cof}(\operatorname{Id}+\boldsymbol{\nabla u}): \nabla \mathbf{v}),
$$

which will be frequently used.

The existence of a solution $(\mathbf{u}, p)$ of Problem 2.1] can be proven as in [12, sections 6.4-6.8]. Following [22, we also assume that

$$
\mathbf{u} \in \mathcal{C}^{2}(\bar{\Omega}), \quad p \in \mathcal{C}^{1}(\bar{\Omega}) .
$$

This regularity is commonly assumed in investigations of nonlinear elasticity, although the assumptions are restrictive. If $\Omega$ is a $\mathcal{C}^{3}$ domain, and the body force $\mathbf{f}$ is sufficiently small in $\mathbf{W}^{1, p}(\Omega)$ with $p>2$, one has $(\mathbf{u}, p) \in \mathbf{W}^{3, p}(\Omega) \times W^{2, p}(\Omega)$, and (2.7) follows by the Sobolev imbedding theorem.

Let $\mathcal{T}_{h}$ be a regular triangulation of $\Omega$ into "quadrilaterals" with mesh parameter $h:=\max _{K \in \mathcal{T}_{h}} h_{K}$. Interelement boundaries are located on straight lines, and elements next to the boundary are transformed/modified to quadrilaterals such that curved boundaries are matched. Moreover, we assume that $\mathcal{T}_{h}$ is obtained by first constructing a triangulation of $\Omega$ with mesh size $2 h$ and then dividing each quadrilateral $\bar{K}$ into four sub-quadrilaterals by the lines connecting the midpoints of the opposite edges of $\bar{K}$. To define the isoparametric elements, let $Q_{k}(K)$ be the space of polynomials of degree $\leq k$ in each variable, and set

$$
\begin{aligned}
& \mathbf{X}_{h}:=\left\{\mathbf{v} \in \mathbf{X} \mid \mathbf{v}_{\left.\right|_{K}} \in Q_{1}(K)^{2} \quad \forall K \in \mathcal{T}_{h}\right\}, \\
& M_{h}:=\left\{q \in M \mid q_{\left.\right|_{K}} \in Q_{0}(\bar{K}) \quad \forall \bar{K} \in \mathcal{T}_{2 h}\right\},
\end{aligned}
$$

for the approximation of the displacement $\mathbf{u}$ and the pressure variable $p$, respectively. In principle, $\left(\mathbf{X}_{h}, M_{h}\right)$ can be any pair of spaces that is stable for the Stokes problem (provided that the interpolation process in Section 4 is adapted); cf. Remark 4.2 .

The finite element approximation of Problem 2.1 reads:

Problem 2.2. Find $\left(\mathbf{u}_{h}, p_{h}\right) \in \mathbf{X}_{h} \times M_{h}$ such that

$$
\begin{aligned}
a\left(\left(\mathbf{u}_{h}, p_{h}\right), \mathbf{v}\right) & =\langle\mathbf{f}, \mathbf{v}\rangle, & & \forall \mathbf{v} \in \mathbf{X}_{h}, \\
b\left(\mathbf{u}_{h}, q\right)-\lambda^{-1}\left(p_{h}, q\right) & =0, & & \forall q \in M_{h} .
\end{aligned}
$$

Note that functions in $M_{h}$ are discontinuous, and the pressure variable $p_{h}$ can be eliminated in finite element computations on an element level. 
2.2. Final assumptions and main problem. Problems 2.1 and 2.2 will be embedded later into one-parameter families of equations. When applying an implicit function theorem, we make use of the derivatives of the nonlinear form $a((\mathbf{u}, p), \mathbf{v})$,

$$
\begin{aligned}
a_{\mathbf{u}}(p ; \mathbf{w}, \mathbf{v}) & :=(\boldsymbol{\nabla} \mathbf{w}, \boldsymbol{\nabla} \mathbf{v})+(p, \operatorname{div}(\operatorname{adj} \boldsymbol{\nabla} \mathbf{w} \mathbf{v})), \\
a_{p}(\mathbf{u} ; \mathbf{v}, q) & :=(q, \operatorname{div}(\operatorname{adj}(\operatorname{Id}+\boldsymbol{\nabla} \mathbf{u}) \mathbf{v})) .
\end{aligned}
$$

The derivatives of $b$ can be expressed in terms of $a_{p}$ and $b$. Moreover, $a_{u}$ is symmetrical in the last two arguments.

Let $(\mathbf{u}, p)$ be the solution of Problem 2.1 and let $a_{\mathbf{u}}(\mathbf{w}, \mathbf{v})$ and $a_{p}(\mathbf{v}, q)$ be abbreviations of $a_{\mathbf{u}}(p ; \mathbf{w}, \mathbf{v})$ and $a_{p}(\mathbf{u} ; \mathbf{v}, q)$, respectively. We consider the following auxiliary linear problem.

Problem 2.3. Given $(\mathbf{F}, G) \in \mathbf{X}^{\prime} \times M^{\prime}$, find $(\mathbf{w}, r) \in \mathbf{X} \times M$ such that

$$
\begin{aligned}
a_{\mathbf{u}}(\mathbf{w}, \mathbf{v})+a_{p}(\mathbf{v}, r) & =\langle\mathbf{F}, \mathbf{v}\rangle, & & \forall \mathbf{v} \in \mathbf{X}, \\
a_{p}(\mathbf{w}, q)-\lambda^{-1}(r, q) & =(G, q), & & \forall q \in M .
\end{aligned}
$$

We define a bilinear form $\mathcal{A}$ as

$$
\mathcal{A}(\mathbf{w}, r ; \mathbf{v}, q):=a_{\mathbf{u}}(\mathbf{w}, \mathbf{v})+a_{p}(\mathbf{v}, r)+a_{p}(\mathbf{w}, q)-\lambda^{-1}(r, q) .
$$

In particular, 2.11 is now rewritten:

$$
\mathcal{A}(\mathbf{w}, r ; \mathbf{v}, q)=\langle\mathbf{F}, \mathbf{v}\rangle+(G, q), \quad \forall(\mathbf{v}, q) \in \mathbf{X} \times M .
$$

Since $\mathcal{A}$ is a bounded operator, it immediately induces a bounded linear operator $\mathcal{L}: \mathbf{X} \times M \rightarrow \mathbf{X}^{\prime} \times M^{\prime}$ by the relation $\mathcal{L}(\mathbf{w}, r)(\mathbf{v}, q)=\mathcal{A}(\mathbf{w}, r ; \mathbf{v}, q)$ for any $(\mathbf{w}, r)$ and $(\mathbf{v}, q) \in \mathbf{X} \times M$. Note that we write $\mathcal{L}(\mathbf{w}, r)(\mathbf{v}, q)$ rather than $(\mathcal{L}(\mathbf{w}, r),(\mathbf{v}, q))$.

For the linear problem (2.11), we have

Theorem 2.4 ([15, Theorem 1]). Assume that $p \in \mathcal{C}^{1}(\bar{\Omega})$ and $\mathbf{u} \in \mathcal{C}^{2}(\bar{\Omega})$.

(1) Let $C_{1}:=\frac{1}{2}\|p\|_{\mathcal{C}^{1}(\bar{\Omega})}^{2}$. Then

$$
\frac{1}{2}\|\nabla \mathbf{v}\|_{0}^{2} \leq a_{\mathbf{u}}(\mathbf{v}, \mathbf{v})+C_{1}\|\mathbf{v}\|_{0}^{2}, \quad \forall \mathbf{v} \in \mathbf{X} .
$$

(2) There exists a constant $C_{2}$ which may depend on $\|\mathbf{u}\|_{\mathcal{C}^{2}(\bar{\Omega})}$ such that

$$
\sup _{\mathbf{v} \in \mathbf{X}} \frac{a_{p}(\mathbf{v}, q)}{\|\mathbf{v}\|_{1}} \geq C_{2}\|q\|_{0}, \quad \forall q \in M .
$$

Theorem 2.4provides only a Gårding inequality for the bilinear form $\mathcal{A}$ in (2.12), and thus the theorem does not exclude the case that Problem 2.3 is not solvable. This is in accordance with the fact that the original problem is nonlinear, and large loads can cause a breakdown of the mechanical system. On the other hand, it is clear that the form $a_{u}(\cdot, \cdot)$ is coercive for small loads and if the solution $(u, p)$ can also be reached on a path without branch points or singularities. Roughly speaking, we have to stay in the region of stability.

After excluding the singularities above and having coercivity in addition to Theorem 2.4 we can verify the following assumption by the general theory of saddle point problems with penalty terms. We recall that the arguments heavily depend on (2.7), and so we prefer the formulation as an assumption.

Assumption R. Given $(\mathbf{F}, G) \in \mathbf{X}^{\prime} \times M^{\prime}$, there exists a unique solution $(\mathbf{w}, r) \in$ $\mathbf{X} \times M$ of Problem 2.3 and

$$
\|\mathbf{w}\|_{1}+\|r\|_{0} \leq C\left(\|\mathbf{F}\|_{-1}+\|G\|_{0}\right) .
$$

Here, $C$ may depend on $\mathbf{u}$ and $p$. 
The assumption above actually implies that the operator $\mathcal{L}$ is an isomorphism. Now we are ready to state our main result.

Theorem 2.5. Let $(\mathbf{u}, p) \in \mathcal{C}^{2}(\bar{\Omega}) \times \mathcal{C}^{1}(\bar{\Omega})$ be the solution of the Problem $[2.1$, and let Assumption $\mathbf{R}$ hold. Then for sufficiently small $h$, there exists a solution $\left(\mathbf{u}_{h}, p_{h}\right) \in \mathbf{X}_{h} \times M_{h}$ of Problem 2.2 in a $\mathbf{W}^{1, \infty}(\Omega) \times L^{\infty}(\Omega)$-neighborhood of $(\mathbf{u}, p)$ such that

$$
\left\|\mathbf{u}-\mathbf{u}_{h}\right\|_{L^{\infty}}+h|\ln h|^{3 / 2}\left(\left\|\nabla\left(\mathbf{u}-\mathbf{u}_{h}\right)\right\|_{L^{\infty}}+\left\|p-p_{h}\right\|_{L^{\infty}}\right) \leq C h^{2}|\ln h|^{2},
$$

where $C$ may depend on $\|\mathbf{u}\|_{\mathcal{C}^{2}(\bar{\Omega})}$ and $\|p\|_{\mathcal{C}^{1}(\bar{\Omega})}$.

This theorem states that the isolated solution can be approximated with quasioptimal error bounds. For large data $\mathbf{f}$, the uniqueness of the solution may be violated; see [12, section 5.8].

Our result improves a similar one in [15, Theorem 2], since the convergence rate presented there is only of order $\mathcal{O}\left(h^{2-\epsilon}\right)$ with $\epsilon>0$.

The main ingredient of our proof is a bound of the regularized Green's functions that will be derived in Section 5. Such kinds of estimates cannot be found in the present literature.

Remark 2.6. Notice that this theorem and its analysis also cover the limit case $\lambda \rightarrow \infty$, i.e., the incompressible material.

Remark 2.7. The error bounds for the displacement with respect to the $\mathbf{W}^{1, \infty}$ norm and the pressure variable with respect to the $L^{\infty}$-norm are the same with the sharp results for the Stokes problem [16, Corollary 5.1 with $k=1$ ]. The $\mathbf{L}^{\infty}$-error bound for the displacement, however, is polluted by an extra factor of $|\ln h|$.

Remark 2.8. Theorem 2.5 is also valid while the finite element spaces are replaced by the $\left(Q_{2}, P_{1}\right)$ pair for $u$ and $p$, respectively, called $9-3$ element by the engineering community [28, 36].

2.3. Embedding Problem 2.2. We will consider a one-parameter family of problems with solutions in a neighborhood of $(\mathbf{u}, p)$. For any $h>0$ we define a meshdependent norm on $\mathbf{W}^{1, \infty}(\Omega) \times L^{\infty}(\Omega)$,

$$
\|\mathbf{v}, q\|_{h}:=\|\mathbf{v}\|_{L^{\infty}}+h|\ln h|^{3 / 2}\left(\|\nabla \mathbf{v}\|_{L^{\infty}}+\|q\|_{L^{\infty}}\right) .
$$

For $\gamma>0$ which will be fixed later, set

$$
U_{\gamma}:=\left\{(\mathbf{v}, q) \in \mathbf{W}^{1, \infty}(\Omega) \times L^{\infty}(\Omega) \mid\|\mathbf{v}-\mathbf{u}, q-p\|_{h} \leq \gamma\right\} .
$$

Next, we introduce a homotopy

$$
\mathcal{H}(t,(\mathbf{w}, q)):[0,1] \times\left(\left(\mathbf{X}_{h} \times M_{h}\right) \cap U_{\gamma}\right) \rightarrow \mathbf{X}_{h}^{\prime} \times M_{h}^{\prime},
$$

between the original nonlinear problem and a linear one. When applied to $(\mathbf{v}, q) \in$ $\mathbf{X}_{h} \times M_{h}$, the operator $\mathcal{H}(t,(\mathbf{w}, r))$ takes the form

$$
\mathcal{H}(t,(\mathbf{w}, r))(\mathbf{v}, q)=\left\{\begin{array}{l}
t[a((\mathbf{w}, r), \mathbf{v})-\langle\mathbf{f}, \mathbf{v}\rangle] \\
+(1-t)\left[a_{\mathbf{u}}(p ; \mathbf{w}-\mathbf{u}, \mathbf{v})+a_{p}(\mathbf{u} ; \mathbf{v}, r-p)\right] \\
t\left[b(\mathbf{w}, q)-\lambda^{-1}(r, q)\right] \\
+(1-t)\left[a_{p}(\mathbf{u} ; \mathbf{w}-\mathbf{u}, q)-\lambda^{-1}(r-p, q)\right] .
\end{array}\right.
$$


$\mathcal{H}(t,(\mathbf{w}, r))$ is continuously differentiable with respect to $(\mathbf{w}, r)$ with derivatives

$$
\mathcal{D}_{(\mathbf{w}, r)} \mathcal{H}(t,(\mathbf{w}, r))((\boldsymbol{\phi}, s),(\mathbf{v}, q))=\left\{\begin{array}{c}
t\left[a_{\mathbf{u}}(r ; \boldsymbol{\phi}, \mathbf{v})+a_{p}(\mathbf{w} ; \mathbf{v}, s)\right] \\
+(1-t)\left[a_{\mathbf{u}}(p ; \boldsymbol{\phi}, \mathbf{v})+a_{p}(\mathbf{u} ; \mathbf{v}, s)\right], \\
t a_{p}(\mathbf{w} ; \boldsymbol{\phi}, q) \\
+(1-t) a_{p}(\mathbf{u} ; \boldsymbol{\phi}, q)-\lambda^{-1}(s, q) .
\end{array}\right.
$$

We will omit the subscript of $\mathcal{D}_{(\mathbf{w}, q)} \mathcal{H}(t,(\mathbf{w}, q))$ when no confusion can occur.

Now we are prepared for the embedding of Problem 2.2 .

Problem 2.9. Find $\left(\mathbf{u}_{h}^{t}, p_{h}^{t}\right) \in\left(\mathbf{X}_{h} \times M_{h}\right) \cap U_{\gamma}$ for some $\gamma>0$ such that

$$
\mathcal{H}\left(t,\left(\mathbf{u}_{h}^{t}, p_{h}^{t}\right)\right)(\mathbf{v}, q)=0, \quad \forall(\mathbf{v}, q) \in \mathbf{X}_{h} \times M_{h} .
$$

Here $t$ is the deformation parameter. The problem is linear for $t=0$, and for $t=1$ we end with the original nonlinear Problem 2.2. In particular, $\left(\mathbf{u}_{h}^{0}, p_{h}^{0}\right)$ can be understood as the Fortin interpolant of $(\mathbf{u}, p)$ in $X_{h} \times M_{h}$ for the mapping given by Problem [2.3 We will exploit the continuity method [19, 31] to prove existence and to derive the error bounds simultaneously.

The linearized expressions are easily separated from the nonlinear variational form by using (2.5) and (2.10)

$$
\begin{aligned}
a\left(\left(\mathbf{u}_{h}^{t},\right.\right. & \left.\left.p_{h}^{t}\right), \mathbf{v}\right)-\langle\mathbf{f}, \mathbf{v}\rangle=a\left(\left(\mathbf{u}_{h}^{t}, p_{h}^{t}\right), \mathbf{v}\right)-a((\mathbf{u}, p), \mathbf{v}) \\
= & \left(\boldsymbol{\nabla}\left(\mathbf{u}_{h}^{t}-\mathbf{u}\right), \mathbf{v}\right)+\left(p_{h}^{t}, \operatorname{div}\left(\operatorname{adj} \boldsymbol{\nabla} \mathbf{u}_{h}^{t} \mathbf{v}\right)\right)-(p, \operatorname{div}(\operatorname{adj} \boldsymbol{\nabla u v})) \\
= & \left(\boldsymbol{\nabla}\left(\mathbf{u}_{h}^{t}-\mathbf{u}\right), \mathbf{v}\right)+\left(p, \operatorname{div}\left(\operatorname{adj} \boldsymbol{\nabla}\left(\mathbf{u}_{h}^{t}-\mathbf{u}\right) \mathbf{v}\right)\right) \\
& +\left(p_{h}^{t}-p, \operatorname{div}(\operatorname{adj} \boldsymbol{\nabla} \mathbf{u} \mathbf{v})\right)+\left(p_{h}^{t}-p, \operatorname{div}\left(\operatorname{adj} \boldsymbol{\nabla}\left(\mathbf{u}_{h}^{t}-\mathbf{u}\right) \mathbf{v}\right)\right) \\
= & a_{\mathbf{u}}\left(p ; \mathbf{u}-\mathbf{u}_{h}^{t}, \mathbf{v}\right)+a_{p}\left(\mathbf{u} ; \mathbf{v}, p-p_{h}^{t}\right)+\left(p_{h}^{t}-p ; \operatorname{div} \operatorname{adj} \boldsymbol{\nabla}\left(\mathbf{u}_{h}^{t}-\mathbf{u}\right) \mathbf{v}\right) .
\end{aligned}
$$

Similarly, by using in addition (2.1), we have

$$
\begin{aligned}
&\left(\operatorname{det}\left(\operatorname{Id}+\boldsymbol{\nabla} \mathbf{u}_{h}^{t}\right)-1, q\right)-\lambda^{-1}\left(p_{h}^{t}, q\right) \\
&=(\operatorname{det}(\operatorname{Id}+\nabla \mathbf{u})-1, q)+\left(\operatorname{Cof}(\operatorname{Id}+\nabla \mathbf{u}): \nabla\left(\mathbf{u}_{h}^{t}-\mathbf{u}\right), q\right) \\
&+\left(\operatorname{det} \boldsymbol{\nabla}\left(\mathbf{u}_{h}^{t}-\mathbf{u}\right), q\right)-\lambda^{-1}\left(p_{h}^{t}, q\right) \\
&= \lambda^{-1}(p, q)+\left(\operatorname{div}\left(\operatorname{adj}(\operatorname{Id}+\boldsymbol{\nabla u})\left(\mathbf{u}_{h}^{t}-\mathbf{u}\right)\right), q\right) \\
&+\left(\operatorname{det} \boldsymbol{\nabla}\left(\mathbf{u}_{h}^{t}-\mathbf{u}\right), q\right)-\lambda^{-1}\left(p_{h}^{t}, q\right) \\
&= a_{p}\left(\mathbf{u} ; \mathbf{u}_{h}^{t}-\mathbf{u}, q\right)-\lambda^{-1}\left(p_{h}^{t}-p, q\right)+\left(\operatorname{det} \boldsymbol{\nabla}\left(\mathbf{u}_{h}^{t}-\mathbf{u}\right), q\right) .
\end{aligned}
$$

We insert these expressions in (2.19) to obtain an equation for the error:

$$
\begin{aligned}
a_{\mathbf{u}}\left(\mathbf{u}-\mathbf{u}_{h}^{t}, \mathbf{v}\right)+a_{p}\left(\mathbf{v}, p-p_{h}^{t}\right) & =t\left(p-p_{h}^{t}, \operatorname{div}\left(\operatorname{adj} \boldsymbol{\nabla}\left(\mathbf{u}-\mathbf{u}_{h}^{t}\right) \mathbf{v}\right)\right), & & \forall \mathbf{v} \in \mathbf{X}_{h}, \\
a_{p}\left(\mathbf{u}-\mathbf{u}_{h}^{t}, q\right)-\lambda^{-1}\left(q, p-p_{h}^{t}\right) & =t\left(\operatorname{det} \boldsymbol{\nabla}\left(\mathbf{u}-\mathbf{u}_{h}^{t}\right), q\right), & & \forall q \in M_{h}
\end{aligned}
$$

When performing the homotopy method, we define a subset of the interval $[0,1]$ by

$$
\begin{aligned}
\Xi_{h}(\mathcal{C}):=\{t \in[0,1] \mid & \text { Problem } 2.9 \text { has a solution }\left(\mathbf{u}_{h}^{t}, p_{h}^{t}\right) \in \mathbf{X}_{h} \times M_{h} \\
& \text { such that } \left.\left\|\mathbf{u}-\mathbf{u}_{h}^{t}, p-p_{h}^{t}\right\|_{h} \leq \mathcal{C} h^{2}|\ln h|^{2}\right\} .
\end{aligned}
$$

The set $\Xi_{h}\left(\mathcal{K}_{1}\right)$ will be shown to be nonvoid, open and closed with respect to the interval $[0,1]$ for sufficiently small $h$ and some $\mathcal{K}_{1}>0$. Hence, it coincides with $[0,1]$. The cornerstones are an a priori estimate and the applicability of an inverse function theorem. They are provided in the following two lemmas that will be proven in Section 6 
Lemma 2.10. Let $(\mathbf{u}, p) \in \mathcal{C}^{2}(\bar{\Omega}) \times \mathcal{C}^{1}(\bar{\Omega})$ be the solution of the Problem [2.1, and assume that Assumption $\mathbf{R}$ holds. Then there exist positive constants $h_{1}$ and $\mathcal{K}_{1}$ with the following property: If $\left(\mathbf{u}_{h}^{t}, p_{h}^{t}\right) \in U_{\gamma}$ is a solution of Problem [2.9, with $\gamma:=2 \mathcal{K}_{1} h^{2}|\ln h|^{2}, 0<h<h_{1}$, and $t \in[0,1]$, then we have

$$
\left(\mathbf{u}_{h}^{t}, p_{h}^{t}\right) \in U_{\gamma / 2} \text {. }
$$

Furthermore, $\left(\mathbf{u}_{h}^{0}, p_{h}^{0}\right) \in U_{\gamma / 2}$, i.e., the inclusion holds for $t=0$.

Actually, we will show a stronger result. It is sufficient to have $\left(\mathbf{u}_{h}^{t}, p_{h}^{t}\right) \in U_{\gamma_{0}}$ for some $\gamma_{0}$ of the order $h|\ln h|^{1 / 2}$ instead of $h^{2}|\ln h|^{2}$.

Lemma 2.11. Let $(\overline{\mathbf{u}}, \bar{p}) \in\left(\mathbf{X}_{h} \times M_{h}\right) \cap U_{\mathcal{K}_{1} h^{2}|\ln h|^{2}}$. If $h$ is sufficiently small, then the operator $\mathcal{D H}(t,(\overline{\mathbf{u}}, \bar{p}))$ is a regular mapping from $\mathbf{X}_{h} \times M_{h}$ into itself for all $t \in[0,1]$.

\section{A PRIORI ESTIMATES AND REgUlarity}

First, we consider the regularity of Problem 2.3

Theorem 3.1. For any $(\mathbf{F}, G) \in \mathbf{L}^{2}(\Omega) \times H^{1}(\Omega)$, the solution $(\mathbf{w}, r)$ of Problem 2.3 belongs to $\mathbf{H}^{2}(\Omega) \times H^{1}(\Omega)$ and

$$
\|\mathbf{w}\|_{2}+\|r\|_{1} \leq C\left(\|\mathbf{F}\|_{0}+\|G\|_{1}\right)
$$

Here $C$ is independent of $\lambda$.

Proof. If we restrict $\lambda$ to a bounded set, a proof of the theorem is essentially included in [15. Lemma 3.5]. The key point is that (2.11) is elliptic in the sense of [1] as was observed by Le Dret [21].

Therefore, we only need to consider Problem 2.3 for large $\lambda$, and we assume that $\lambda \geq \lambda_{0}$, with $\lambda_{0}$ to be specified later. We consider an auxiliary problem without penalty term. Find $\left(\mathbf{w}_{1}, r_{1}\right) \in \mathbf{X} \times M$ such that

$$
\begin{array}{lll}
a_{\mathbf{u}}\left(\mathbf{w}_{1}, \mathbf{v}\right)+a_{p}\left(\mathbf{v}, r_{1}\right) & =\langle\mathbf{F}, \mathbf{v}\rangle & \forall \mathbf{v} \in \mathbf{X}, \\
a_{p}\left(\mathbf{w}_{1}, q\right) & =(G, q) & \forall q \in M .
\end{array}
$$

In view of [15, Lemma 3.5], we have

$$
\left\|\mathbf{w}_{1}\right\|_{2}+\left\|r_{1}\right\|_{1} \leq C_{3}\left(\|\mathbf{F}\|_{0}+\|G\|_{1}\right) .
$$

Next we define a mapping $T$ from $H^{1}(\Omega)$ into itself. Given $r$, let $\operatorname{Tr}:=\bar{r}$ be defined by

$$
\begin{aligned}
& a_{\mathbf{u}}(\overline{\mathbf{w}}, \mathbf{v})+a_{p}(\mathbf{v}, \bar{r})=\langle\mathbf{F}, \mathbf{v}\rangle \quad \forall \mathbf{v} \in \mathbf{X}, \\
& a_{p}(\overline{\mathbf{w}}, q) \quad=\left(G+\lambda^{-1} r, q\right) \quad \forall q \in M .
\end{aligned}
$$

Given $r_{1}, r_{2} \in H^{1}(\Omega)$, it follows from (3.3) that $\left\|\bar{r}_{1}-\bar{r}_{2}\right\|_{1} \leq \lambda^{-1} C_{3}\left\|r_{1}-r_{2}\right\|_{1} \leq$ $\frac{1}{2}\left\|r_{1}-r_{2}\right\|_{1}$ whenever $\lambda \geq \lambda_{0}:=2 C_{3}$. Hence, $T$ is a contraction and it has a fixed point $r \in H^{1}(\Omega)$. Let $\mathbf{w}:=\overline{\mathbf{w}}$ be the other component of the solution with this $r$. From

$$
\|\mathbf{w}\|_{2}+\|r\|_{1} \leq C_{3}\left(\|\mathbf{F}\|_{0}+\|G\|_{1}\right)+\frac{1}{2}\|r\|_{1},
$$

we conclude that (3.1) holds with $C:=2 C_{3}$, and the proof is complete.

A duality argument [24], yields the following fact. 
Lemma 3.2. Let $(\mathbf{F}, G) \in \mathbf{H}^{2}(\Omega)^{\prime} \times H^{1}(\Omega)^{\prime}$. Then the solution $(\mathbf{w}, r)$ of Problem 2.3 belongs to $\mathbf{L}^{2}(\Omega) \times H^{-1}(\Omega)$ and

$$
\|\mathbf{w}\|_{0}+\|r\|_{-1} \leq C\left(\|\mathbf{F}\|_{-2}+\|G\|_{-1}\right) .
$$

Proof. Given $(\phi, s) \in \mathbf{L}^{2}(\Omega) \times \mathbf{H}_{0}^{1}(\Omega)$, consider the auxiliary problem: Find $(\overline{\mathbf{w}}, \bar{r}) \in$ $\mathbf{X} \times M$ such that

$$
\mathcal{A}(\mathbf{v}, q ; \overline{\mathbf{w}}, \bar{r})=(\phi, \mathbf{v})+(s, q), \quad \forall(\mathbf{v}, q) \in \mathbf{X} \times M .
$$

Theorem 3.1 implies that $\|\overline{\mathbf{w}}\|_{2}+\|\bar{r}\|_{1} \leq C\left(\|\phi\|_{0}+\|s\|_{1}\right)$. By setting $\mathbf{v}:=\mathbf{w}$ and $q:=r$ in (3.5), it follows that

$$
\begin{aligned}
|(\mathbf{w}, \boldsymbol{\phi})+(r, s)| & =|\mathcal{A}(\mathbf{w}, r ; \overline{\mathbf{w}}, \bar{r})|=|\langle\mathbf{F}, \overline{\mathbf{w}}\rangle+(G, \bar{r})| \\
& \leq\left(\|\mathbf{F}\|_{-2}+\|G\|_{-1}\right)\left(\|\overline{\mathbf{w}}\|_{2}+\|\bar{r}\|_{1}\right) \\
& \leq C\left(\|\mathbf{F}\|_{-2}+\|G\|_{-1}\right)\left(\|\phi\|_{0}+\|s\|_{1}\right),
\end{aligned}
$$

which implies the bounds of $\|\mathbf{w}\|_{0}$ and $\|r\|_{-1}$ as stated in (3.4).

To achieve $L^{\infty}$-estimates, we consider error estimates for weighted Sobolev norms with the weight function

$$
\sigma(\mathbf{x}):=\left(\left|\mathbf{x}-\mathbf{x}_{0}\right|^{2}+\theta^{2}\right)^{1 / 2}, \quad \mathbf{x}, \mathbf{x}_{0} \in \Omega .
$$

Here $\theta=K h$, with $K \geq 1$ being a constant to be specified later. For $\alpha \in \mathbb{R}$ and $k$ a nonnegative integer, we define the weighted semi-norms

$$
\left\|\mathcal{D}^{k} q\right\|_{\sigma^{\alpha}}^{2}:=\sum_{|\beta|=k} \int_{\Omega}\left|\partial^{\beta} q(\mathbf{x})\right|^{2} \sigma(\mathbf{x})^{\alpha} d \mathbf{x}, \quad q \in H^{k}(\Omega)
$$

The same notation will be used for vector-valued functions. We list some properties of $\sigma$ (see [9, p. 170] for more general cases):

$$
\begin{array}{rlrl}
\max _{\mathbf{x} \in K} \sigma(\mathbf{x}) & \leq C \min _{\mathbf{x} \in K} \sigma(\mathbf{x}), & & \forall K \in \mathcal{T}_{h}, \\
\left|\mathcal{D}^{j} \sigma(\mathbf{x})^{\alpha}\right| \leq C(j, \alpha) \sigma^{\alpha-j}(\mathbf{x}), & & \forall \mathbf{x} \in \Omega, \alpha \in \mathbb{R} .
\end{array}
$$

By [11, Theorem 3.3.2], if $\theta$ is small enough,

$$
\int_{\Omega} \sigma^{-(2+\alpha)}(\mathbf{x}) d \mathbf{x} \leq \begin{cases}C \theta^{-\alpha}, & \text { for } \alpha>0 \\ C|\ln \theta|, & \text { for } \alpha=0 .\end{cases}
$$

Theorem 3.3. For any $(\mathbf{F}, G) \in \mathbf{L}^{2}(\Omega) \times H^{1}(\Omega)$, the solution $(\mathbf{w}, r)$ of Problem 2.3 satisfies

$$
\begin{aligned}
\left\|\mathcal{D}^{2} \mathbf{w}\right\|_{\sigma^{2}}+\|\nabla r\|_{\sigma^{2}} & \leq C \theta\left(\|\mathbf{F}\|_{0}+\|G\|_{1}\right) \\
& +C\left(\|\mathbf{F}\|_{\sigma^{2}}+\|\nabla G\|_{\sigma^{2}}+\|\mathbf{F}\|_{-1}+\|G\|_{0}\right) .
\end{aligned}
$$

Proof. As in [11, Chapter 3, Section 3.3], let $\mu_{j}=x^{j}-x_{0}^{j}(j=1,2)$. From $\mu_{j} \boldsymbol{\nabla} r=\boldsymbol{\nabla}\left(\mu_{j} r\right)-\left(\boldsymbol{\nabla} x_{j}\right) r$ and a similar formula for $\mathbf{w}$ it follows that

$$
\left\|\mu_{j} \mathcal{D}^{2} \mathbf{w}\right\|_{0}+\left\|\mu_{j} \boldsymbol{\nabla} r\right\|_{0} \leq C\left(\left\|\mathcal{D}^{2}\left(\mu_{j} \mathbf{w}\right)\right\|_{0}+\left\|\boldsymbol{\nabla}\left(\mu_{j} r\right)\right\|_{0}+\|\boldsymbol{\nabla} \mathbf{w}\|_{0}+\|r\|_{0}\right) .
$$

We only need to establish a bound of the first two terms on the right-hand side since the other terms are covered by (2.15). Observe that the strong form of Problem 2.3 reads

$$
\begin{aligned}
-\Delta \mathbf{w}-(\operatorname{adj} \nabla \mathbf{w}) \boldsymbol{\nabla} p-\operatorname{Cof}(\operatorname{Id}+\boldsymbol{\nabla u}) \boldsymbol{\nabla} r & =\mathbf{F}, \\
\operatorname{adj}(\operatorname{Id}+\boldsymbol{\nabla u}): \boldsymbol{\nabla} \mathbf{w}-\lambda^{-1} r & =G .
\end{aligned}
$$


A simple computation yields

$$
\begin{aligned}
-\triangle\left(\mu_{j} \mathbf{w}\right)-\left(\operatorname{adj} \nabla\left(\mu_{j} \mathbf{w}\right)\right) \boldsymbol{\nabla} p-\operatorname{Cof}(\operatorname{Id}+\nabla \mathbf{u}) \boldsymbol{\nabla}\left(\mu_{j} r\right) & =\overline{\mathbf{F}}, \\
\operatorname{adj}(\operatorname{Id}+\nabla \mathbf{u}): \nabla\left(\mu_{j} \mathbf{w}\right)-\lambda^{-1} \mu_{j} r & =\bar{G},
\end{aligned}
$$

where the modified right-hand sides differ from the original ones only by terms of lower order

$$
\begin{aligned}
& \overline{\mathbf{F}}=\mu_{j} \mathbf{F}-2 \boldsymbol{\nabla} \mu_{j} \boldsymbol{\nabla} \mathbf{w}-\boldsymbol{\nabla} p \boldsymbol{\nabla} \mu_{j}^{\perp} \otimes \mathbf{w}^{\perp}-\operatorname{Cof}(\mathrm{Id}+\nabla \mathbf{u}) \nabla \mu_{j} r, \\
& \bar{G}=\mu_{j} G+\operatorname{adj}(\operatorname{Id}+\nabla \mathbf{u}): \mathbf{w} \otimes \nabla \mu_{j} .
\end{aligned}
$$

By virtue of Theorem 3.1 and Assumption $\mathbf{R}$ it follows that

$$
\begin{aligned}
\left\|\mathcal{D}^{2}\left(\mu_{j} \mathbf{w}\right)\right\|_{0}+\left\|\boldsymbol{\nabla}\left(\mu_{j} r\right)\right\|_{0} & \leq C\left(\left\|\mu_{j} \mathbf{F}\right\|_{0}+\left\|\mu_{j} \boldsymbol{\nabla} G\right\|_{0}+\|\boldsymbol{\nabla} \mathbf{w}\|_{0}+\|r\|_{0}\right) \\
& \leq C\left(\left\|\mu_{j} \mathbf{F}\right\|_{0}+\left\|\mu_{j} \boldsymbol{\nabla} G\right\|_{0}+\|\mathbf{F}\|_{-1}+\|G\|_{0}\right) .
\end{aligned}
$$

Next,

$$
\left\|\mathcal{D}^{2} \mathbf{w}\right\|_{\sigma^{2}}^{2}=\theta^{2}\left\|\mathcal{D}^{2} \mathbf{w}\right\|_{0}^{2}+\sum_{j=1}^{2}\left\|\mu_{j} \mathcal{D}^{2} \mathbf{w}\right\|_{0}^{2} .
$$

Combining this with (3.12) and the analogous estimates of $\nabla r$, we obtain (3.11).

Proceeding along the same line and using Lemma 3.2, we obtain

Lemma 3.4. For any $(\mathbf{F}, G) \in \mathbf{L}^{2}(\Omega) \times H^{1}(\Omega)$, the solution $(\mathbf{w}, r)$ of Problem 2.3 satisfies

$$
\begin{aligned}
\|\boldsymbol{\nabla} \mathbf{w}\|_{\sigma^{2}}+\|r\|_{\sigma^{2}} & \leq C \theta\left(\|\mathbf{F}\|_{-1}+\|G\|_{0}\right) \\
& +C\left(\sum_{j=1}^{2}\left\|\mu_{j} \mathbf{F}\right\|_{-1}+\|G\|_{\sigma^{2}}\right)+C\left(\|\mathbf{F}\|_{-2}+\|G\|_{-1}\right) .
\end{aligned}
$$

\section{An interpolant of Clément type}

In this section, we provide some approximation properties of an interpolant of Clément type. Consider a macroelement $\mathcal{M}$ of $\mathcal{T}_{2 h}$; see Figure 10 Denote its four edges by $\mathcal{E}_{i}(1 \leq i \leq 4)$ and the midpoint of $\mathcal{E}_{i}$ by $a_{i}$. Let $b_{0}$ be the center of $\mathcal{M}$, and let $b_{i}(i=1,2,3,4)$ be the corner nodes labeled anticlockwise from the lower-left corner. The four corresponding squares are denoted by $K_{i}(i=1,2,3,4)$.

We start with the Clément type interpolation operator $R_{h}$ defined in [3]. It will be modified following [4] in order to preserve the integrals over the edges. If we modify an interpolant $v_{h}$ at the midpoints $a_{1}, a_{2}, a_{3}$, and $a_{4}$, only the change of $v_{h}\left(a_{i}\right)$ will contribute to $\int_{\mathcal{E}_{i}} v_{h} d s$. In this way we obtain an interpolation operator

$$
\Pi_{h}: H^{1}(\Omega) \rightarrow\left\{v \in H^{1}(\Omega) \mid v_{\left.\right|_{K}} \in Q_{1}(K) \forall K \in \mathcal{T}_{h}\right\}
$$

that is given by

$$
\begin{gathered}
\left(\Pi_{h}\right)_{\left.\right|_{i}}: H^{1}(\mathcal{M}) \rightarrow Q_{1}\left(K_{i}\right), \quad i=1,2,3,4, \\
\Pi_{h} v\left(b_{i}\right):=R_{h} v\left(b_{i}\right), \quad i=0,1,2,3,4, \\
\int_{\mathcal{E}_{i}}\left(v-\Pi_{h} v\right) d \mathbf{s}=0, \quad 1 \leq i \leq 4 .
\end{gathered}
$$




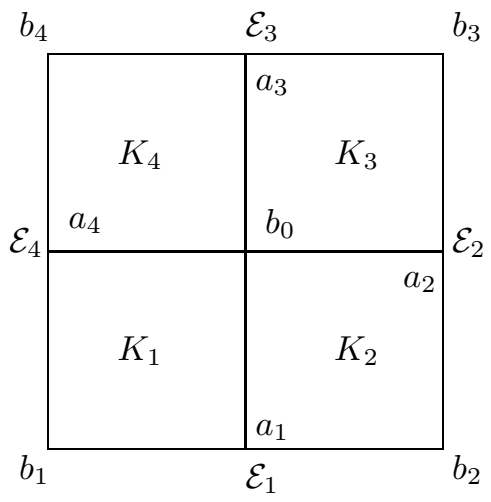

FiguRE 1. Macroelement $\mathcal{M}$

The interpolation process preserves the nullity of traces due to $(4.1)_{2}$ and 4.1$)_{3}$. For a vector function $\mathbf{v}=\left(v_{1}, v_{2}\right) \in \mathbf{H}_{0}^{1}(\Omega)$, we define the interpolant $\Pi_{h} \mathbf{v}$ by the components:

$$
\Pi_{h} \mathbf{v}:=\left(\Pi_{h} v_{1}, \Pi_{h} v_{2}\right) .
$$

Similarly $\mathbf{R}_{h} \mathbf{v}:=\left(R_{h} v_{1}, R_{h} v_{2}\right)$.

We note that the construction of a similar operator in 4 is based on a Ritz projector. The quasi-local character of the operator (4.1) enables us, however, to establish the estimates (4.6) and (4.7) below.

Theorem 4.1. The interpolation operator $\Pi_{h}$ defined by (4.1) and (4.2) has the following properties:

(1) For any matrix $\mathbf{A}$ whose elements are piecewise constant on each $K$ of $\mathcal{T}_{2 h}$, we have

$$
\sum_{\mathcal{M} \in \mathcal{T}_{2 h}} \int_{\mathcal{M}} \operatorname{div}\left(\mathbf{A}\left(\mathbf{v}-\mathbf{\Pi}_{h} \mathbf{v}\right)\right) q d \mathbf{x}=0, \quad \forall q \in M_{h}
$$

(2) There exists a constant $C$ such that

$$
\begin{aligned}
& \left\|\mathcal{D}^{k}\left(\mathbf{v}-\Pi_{h} \mathbf{v}\right)\right\|_{L^{p}} \leq C h^{j-k}\left\|\mathcal{D}^{j} \mathbf{v}\right\|_{L^{p}}, \quad k=0,1 \text { and } j=1,2, \quad p \in[1, \infty], \\
& \left\|\mathcal{D}^{k}\left(\mathbf{v}-\mathbf{\Pi}_{h} \mathbf{v}\right)\right\|_{\sigma^{2}} \leq C h^{j-k}\left\|\mathcal{D}^{j} \mathbf{v}\right\|_{\sigma^{2}}, \quad k=0,1 \text { and } j=1,2 .
\end{aligned}
$$

(3) There exists a constant $C$ such that

$$
\left\|\boldsymbol{\nabla}\left(\sigma^{2} \mathbf{v}-\boldsymbol{\Pi}_{h}\left(\sigma^{2} \mathbf{v}\right)\right)\right\|_{\sigma^{-2}} \leq C h\|\mathbf{v}\|_{\sigma^{-2}}+C h\|\nabla \mathbf{v}\|_{0}, \quad \forall \mathbf{v} \in \mathbf{X}_{h} .
$$

(4) There exists a constant $C$ such that for all $p \in[1, \infty]$

$$
\left\|\boldsymbol{\nabla} \Pi_{h} \mathbf{v}\right\|_{L^{p}} \leq C\|\boldsymbol{\nabla} \mathbf{v}\|_{L^{p}} \text { and }\left\|\boldsymbol{\nabla} \boldsymbol{\Pi}_{h} \mathbf{v}\right\|_{\sigma^{2}} \leq C\|\boldsymbol{\nabla} \mathbf{v}\|_{\sigma^{2}} .
$$

Here and in the proof, $\mathcal{D}^{2} \mathbf{v}$ is understood in a piecewise manner for any $\mathbf{v} \in \mathbf{X}_{h}$, and so are $\left\|\mathcal{D}^{2} \mathbf{v}\right\|_{L^{p}}$ and $\left\|\mathcal{D}^{2} \mathbf{v}\right\|_{\sigma^{2}}$.

A similar bound for the first part of (4.7) with $p=2$ is included in [4, Theorem 3.1]. The main ingredient of the following proof is the scaled trace inequality as well as an error estimate of $\mathbf{R}_{h}$. 
Proof. (1) Let $\mathbf{A}$ be a matrix whose elements are constant on each $\mathcal{M}$ in $\mathcal{T}_{2 h}$. By (4.1) 3 we obtain for the normal components $\int_{\mathcal{E}_{i}} \mathbf{A}\left(\boldsymbol{\Pi}_{h} \mathbf{v}-\mathbf{v}\right) \cdot \mathbf{n} d \mathbf{s}=0$ on each edge $\mathcal{E}_{i}$ of the $2 h$-grid. Gauss' theorem yields now (4.3).

(2) We restrict ourselves to $p<\infty$ since the case $p=\infty$ follows the same lines.

Referring to Figure 1, we select $K_{1}$ as a typical element in $\mathcal{T}_{h}$. A simple scaling argument yields

$$
\int_{K_{1}}\left|\left(\boldsymbol{\Pi}_{h}-\mathbf{R}_{h}\right) \mathbf{v}\right|^{p} d \mathbf{x} \leq C h_{K_{1}}^{2} \sum_{i=1}^{4}\left|\left(\boldsymbol{\Pi}_{h}-\mathbf{R}_{h}\right) \mathbf{v}\left(x_{i}\right)\right|^{p},
$$

where $x_{1}=b_{1}, x_{2}=a_{1}, x_{3}=b_{0}, x_{4}=a_{4}$. Since $\left(\boldsymbol{\Pi}_{h}-\mathbf{R}_{h}\right) \mathbf{v}$ vanishes at $b_{0}$ and $b_{1}$, we obtain contributions only from $a_{1}$ and $a_{4}$. Similar estimates hold for the integrals on $K_{2}, K_{3}$ and $K_{4}$. Summing up, we have on the macroelement $\mathcal{M} \in \mathcal{T}_{2 h}$,

$$
\int_{\mathcal{M}}\left|\left(\boldsymbol{\Pi}_{h}-\mathbf{R}_{h}\right) \mathbf{v}\right|^{p} d \mathbf{x} \leq C h_{\mathcal{M}}^{2} \sum_{i=1}^{4}\left|\left(\boldsymbol{\Pi}_{h}-\mathbf{R}_{h}\right) \mathbf{v}\left(a_{i}\right)\right|^{p} .
$$

Next, since $\left(\boldsymbol{\Pi}_{h}-\mathbf{R}_{h}\right) \mathbf{v}$ is piecewise linear on $\mathcal{E}_{i}$ and vanishes at the vertices of $\mathcal{M}$, we have in view of $(4.1)_{3}$,

$$
\left(\boldsymbol{\Pi}_{h}-\mathbf{R}_{h}\right) \mathbf{v}\left(a_{i}\right)=\frac{1}{\left|\mathcal{E}_{i}\right|} \int_{\mathcal{E}_{i}}\left(\boldsymbol{\Pi}_{h}-\mathbf{R}_{h}\right) \mathbf{v} d \mathbf{s}=\frac{1}{\left|\mathcal{E}_{i}\right|} \int_{\mathcal{E}_{i}}\left(\mathbf{v}-\mathbf{R}_{h} \mathbf{v}\right) d \mathbf{s} .
$$

Combining (4.8) with (4.9) and using the scaled trace inequality 20 Theorem 1.5.1.10] and Hölder's inequality, we obtain for any $j=1,2$,

$$
\begin{aligned}
\int_{\mathcal{M}} \mid\left(\boldsymbol{\Pi}_{h}\right. & \left.-\mathbf{R}_{h}\right)\left.\mathbf{v}\right|^{p} d \mathbf{x} \leq C h_{\mathcal{M}} \sum_{i=1}^{4} \int_{\mathcal{E}_{i}}\left|\mathbf{v}-\mathbf{R}_{h} \mathbf{v}\right|^{p} d \mathbf{s} \\
& \leq C h_{\mathcal{M}}\left(h_{\mathcal{M}}^{-1} \int_{\mathcal{M}}\left|\mathbf{v}-\mathbf{R}_{h} \mathbf{v}\right|^{p} d \mathbf{x}+h_{\mathcal{M}}^{p-1} \int_{\mathcal{M}}\left|\nabla\left(\mathbf{v}-\mathbf{R}_{h} \mathbf{v}\right)\right|^{p} d \mathbf{x}\right) \\
& \leq C h_{\mathcal{M}}^{j p} \int_{\omega(\mathcal{M})}\left|\mathcal{D}^{j} \mathbf{v}\right|^{p} d \mathbf{x},
\end{aligned}
$$

where $\omega(\mathcal{M}):=\left\{\mathcal{M}^{\prime} \in \mathcal{T}_{2 h} \mid \mathcal{M}^{\prime} \cap \mathcal{M} \neq \emptyset\right\}$. An application of the inverse inequality leads to

$$
\left\|\boldsymbol{\nabla}\left(\boldsymbol{\Pi}_{h}-\mathbf{R}_{h}\right) \mathbf{v}\right\|_{L^{p}(\mathcal{M})} \leq C h_{\mathcal{M}}^{(j-1) p}\left\|\mathcal{D}^{j} \mathbf{v}\right\|_{L^{p}(\omega(\mathcal{M}))}, \quad j=1,2 .
$$

Summing up all $\mathcal{M} \in \mathcal{T}_{2 h}$ in (4.10) and (4.11), we obtain

$$
\left\|\mathcal{D}^{k}\left(\boldsymbol{\Pi}_{h} v-\mathbf{R}_{h} \mathbf{v}\right)\right\|_{L^{p}} \leq C h^{j-k}\left\|\mathcal{D}^{j} \mathbf{v}\right\|_{L^{p}}, \quad k=0,1 \text { and } j=1,2, \quad p \in[1, \infty) .
$$

This inequality together with the well-known estimates for $\mathbf{R}_{h}[3$, namely

$$
\left\|\mathcal{D}^{k}\left(\mathbf{v}-\mathbf{R}_{h} \mathbf{v}\right)\right\|_{L^{p}} \leq C h^{j-k}\left\|\mathcal{D}^{j} \mathbf{v}\right\|_{L^{p}}, \quad k=0,1 \text { and } j=1,2, \quad p \in[1, \infty),
$$

leads to (4.4) for all $j$ and $k$, which together with (3.8) immediately yields (4.5).

(3) Since the triangulation $\mathcal{T}_{h}$ is quasi-uniform, we can derive in the same way as 3.8

$$
\max _{\mathbf{x} \in K} \sigma(\mathbf{x}) \leq C \min _{\mathbf{x} \in \omega(\mathcal{M})} \sigma(\mathbf{x}), \quad \forall K \subset \mathcal{M} \in \mathcal{T}_{2 h}
$$


We recall (4.11) for $p=2$, i.e., $\left\|\boldsymbol{\nabla}\left(\mathbf{w}-\boldsymbol{\Pi}_{h} \mathbf{w}\right)\right\|_{L^{2}(K)} \leq C h_{\mathcal{M}}\left\|\mathcal{D}^{2} \mathbf{w}\right\|_{L^{2}(w(\mathcal{M}))}$, for $K \subset \mathcal{M} \in \mathcal{T}_{2 h}$. Together with (4.12) we have

$$
\int_{K} \sigma(\mathbf{x})^{-2}\left|\nabla\left(\mathbf{w}-\Pi_{h} \mathbf{w}\right)\right| d \mathbf{x} \leq C h_{\mathcal{M}}^{2} \int_{\omega(\mathcal{M})} \sigma(\mathbf{x})^{-2}\left|\mathcal{D}^{2} \mathbf{w}\right|^{2} d \mathbf{x} .
$$

Summing up for all $K \in \mathcal{T}_{h}$ gives

$$
\left\|\boldsymbol{\nabla}\left(\mathbf{w}-\boldsymbol{\Pi}_{h} \mathbf{w}\right)\right\|_{\sigma^{-2}} \leq C h\left\|\mathcal{D}^{2}\left(\sigma^{2} \mathbf{w}\right)\right\|_{\sigma^{-2}} .
$$

For any $\mathbf{v} \in \mathcal{T}_{h}$, letting $\mathbf{w}$ be $\sigma^{2} \mathbf{v}$ in the above inequality, we get

$\left\|\boldsymbol{\nabla}\left(\sigma^{2} \mathbf{v}-\boldsymbol{\Pi}_{h}\left(\sigma^{2} \mathbf{v}\right)\right)\right\|_{\sigma^{-2}} \leq C h\left\|\mathcal{D}^{2}\left(\sigma^{2} \mathbf{v}\right)\right\|_{\sigma^{-2}} \leq C\left(h\|\mathbf{v}\|_{\sigma^{-2}}+h\|\nabla \mathbf{v}\|_{0}+h^{2}\left\|\mathcal{D}^{2} \mathbf{v}\right\|_{0}\right)$.

An inverse inequality for finite element spaces applied to the last term yields (4.6).

(4) Finally, by applying (4.4) and (4.5) with $k=j=1$ and using the triangle inequality, we obtain the bound (4.7).

Remark 4.2. The property (4.3) is similar to the key assumption in [16 (1.8)], where the assumption was written for $\mathbf{A}$ being the identity matrix. The essential point is that the pair $\left(\mathbf{X}_{h}, M_{h}\right)$ is stable for the Stokes problem and there is a locally constructed operator with properties like the Fortin interpolant.

Similar constructions can be performed for other pairs of finite element spaces; see [16, Section 5]. In particular the aforementioned $\left(Q_{2}, P_{1}\right)$ element (see Remark [2.8) is easily treated by combining the procedure above with techniques in [7] and [10].

A standard scaling argument together with an inverse inequality yields the following approximation and superapproximation properties that refer to the other function in the variational problem.

Theorem 4.3. Let $\mathcal{Q}_{h}$ be the $L^{2}$-projection onto $M_{h}$.

(1) There exists a constant $C$ such that

$$
\begin{aligned}
\left\|q-\mathcal{Q}_{h} q\right\|_{L^{p}} & \leq C h\|\nabla q\|_{L^{p}}, \quad \forall p \in[1, \infty], \\
\left\|q-\mathcal{Q}_{h} q\right\|_{\sigma^{2}} & \leq C h\|\nabla q\|_{\sigma^{2}} .
\end{aligned}
$$

(2) $\mathcal{Q}_{h}$ admits the superapproximation property

$$
\left\|\sigma^{2} q-\mathcal{Q}_{h}\left(\sigma^{2} q\right)\right\|_{\sigma^{-2}} \leq C h\|q\|_{0}, \quad \forall q \in M_{h} .
$$

\section{Regularized Green's Functions and $L^{\infty}$-estimates}

The proof of Lemma 2.10 will be based on $L^{\infty}$-estimates of the terms on the right-hand side of (2.20). They will be obtained by the technique proposed by Frehse and Rannacher [18 and refer to the regularized Green's functions and the weighted Sobolev norm estimates introduced by Natterer [25]. Our approach is similar to that in [16]; the concept is even more transparent in the scalar case [9] Chapter 7]. In the present situation, there is, however, an extra difficulty. We have only a Gärding inequality instead of the usual coercivity of $a_{\mathbf{u}}(\cdot, \cdot)$. Thus the error bounds with respect to the energy norm are interwoven with an $L^{2}$-estimate, and the latter requires an appropriate duality argument.

Lemma 2.10 could also be proven by weighted Sobolev norm estimates as in [15], but only an order of $h^{2-\epsilon}$ would be achieved in (2.16). Moreover, the estimates for the regularized Green's functions are of independent interest. 
We start with a general argument following [18]. Let $\chi=\chi_{i}(1 \leq i \leq 3)$ be a piecewise polynomial, and let $|\chi|$ attain its maximum at some $\mathbf{x}_{i} \in K_{i}$. Since the mesh $\mathcal{T}_{h}$ is regular, we can find a ball $\mathcal{B}_{i} \subset K_{i}$ with radius $\alpha h$, such that its center $\mathbf{y}_{i}$ satisfies $\left|\mathbf{x}_{i}-\mathbf{y}_{i}\right| \leq C \alpha h$. Here $\alpha$ is assumed to be sufficiently small depending on the shape parameter of the triangulation $\mathcal{T}_{h}$. Next, let $\delta_{i} \in \mathcal{C}_{0}^{\infty}(\Omega)$ satisfy

$$
\begin{aligned}
\operatorname{supp} \delta_{i} & \subset \mathcal{B}_{i}, \\
\int_{\Omega} \delta_{i} d \mathbf{x} & =1, \quad \delta_{i} \geq 0, \\
\left\|\mathcal{D}^{j} \delta_{i}\right\|_{L^{\infty}} & \leq C h^{-2-j}, \quad j=0,1 .
\end{aligned}
$$

The mean value theorem, combined with (5.2), yields

$$
\int_{\Omega} \chi \delta d \mathbf{x}=\chi(\mathbf{z}) \quad \text { for some } \quad \mathbf{z} \in \mathcal{B}_{i} \text {. }
$$

Using an inverse inequality for polynomials leads to

$$
\|\chi\|_{L^{\infty}(\Omega)}=\left|\chi\left(\mathbf{x}_{i}\right)\right| \leq|\chi(\mathbf{z})|+C \alpha h\|\nabla \chi\|_{L^{\infty}\left(K_{1}\right)} \leq C \alpha\|\chi\|_{L^{\infty}(\Omega)}+\left|\int_{\Omega} \chi \delta d \mathbf{x}\right| .
$$

Choosing $\alpha$ such that $C \alpha=1 / 2$, we may rewrite the previous inequality as

$$
\|\chi\|_{L^{\infty}(\Omega)} \leq 2\left|\int_{\Omega} \chi \delta d \mathbf{x}\right|
$$

Therefore the required bound can be obtained from an estimate of a functional.

We define three families of regularized Green's functions and describe simultaneously their relation to $L^{\infty}$-estimates. By duality, first their $L^{1}$-norms enter what in turn will be bounded by weighted $L^{2}$-norms.

Lemma 5.1. Let $\left(\mathbf{G}_{1}, \zeta_{1}\right) \in \mathbf{X} \times M$ and the corresponding regularized Green's functions $\left(\mathbf{G}_{1}^{h}, \zeta_{1}^{h}\right) \in \mathbf{X}_{h} \times M_{h}$ satisfy

$$
\begin{aligned}
& \mathcal{A}\left(\mathbf{G}_{1}, \zeta_{1} ; \mathbf{v}, q\right)=\left(\boldsymbol{\delta}_{1}, \mathbf{v}\right), \quad \forall(\mathbf{v}, q) \in \mathbf{X} \times M \\
& \mathcal{A}\left(\mathbf{G}_{1}^{h}, \zeta_{1}^{h} ; \mathbf{v}, q\right)=\left(\boldsymbol{\delta}_{1}, \mathbf{v}\right), \quad \forall(\mathbf{v}, q) \in \mathbf{X}_{h} \times M_{h}
\end{aligned}
$$

respectively. Here $\boldsymbol{\delta}_{1}=\left(\delta_{1}, 0\right)$ or $\boldsymbol{\delta}_{1}=\left(0, \delta_{1}\right)$. Then we have

$$
\begin{aligned}
\left\|\mathbf{u}-\mathbf{u}_{h}^{t}\right\|_{L^{\infty}} & \leq 2\left\|\mathbf{u}-\boldsymbol{\Pi}_{h} \mathbf{u}\right\|_{L^{\infty}}+C|\ln h|^{1 / 2}\left(\left\|\nabla\left(\mathbf{u}-\boldsymbol{\Pi}_{h} \mathbf{u}\right)\right\|_{L^{\infty}}+\left\|p-\mathcal{Q}_{h} p\right\|_{L^{\infty}}\right) \\
& \times\left(\left\|\boldsymbol{\nabla}\left(\mathbf{G}_{1}-\mathbf{G}_{1}^{h}\right)\right\|_{\sigma_{1}^{2}}+\left\|\zeta_{1}-\zeta_{1}^{h}\right\|_{\sigma_{1}^{2}}\right) \\
& +C t|\ln h|^{1 / 2}\left\|\boldsymbol{\nabla}\left(\mathbf{u}-\mathbf{u}_{h}^{t}\right)\right\|_{L^{\infty}}^{2}\left\|\zeta_{1}^{h}\right\|_{\sigma_{1}^{2}} \\
& +C t|\ln h|^{1 / 2}\left\|\boldsymbol{\nabla}\left(\mathbf{u}-\mathbf{u}_{h}^{t}\right)\right\|_{L^{\infty}}\left\|p-p_{h}^{t}\right\|_{L^{\infty}}\left\|\nabla \mathbf{G}_{1}^{h}\right\|_{\sigma_{1}^{2}} .
\end{aligned}
$$

Proof. It is enough to establish a bound of $\left\|\mathbf{\Pi}_{h} \mathbf{u}-\mathbf{u}_{h}^{t}\right\|_{L^{\infty}}$. To this end, let $\mathbf{x}_{1} \in \Omega$ be a point where the maximum is attained:

$$
\left\|\boldsymbol{\Pi}_{h} \mathbf{u}-\mathbf{u}_{h}^{t}\right\|_{L^{\infty}}=\max _{1 \leq i \leq 2}\left|\left(\left(\boldsymbol{\Pi}_{h} \mathbf{u}\right)^{i}-\mathbf{u}_{h}^{t, i}\right)\left(\mathbf{x}_{1}\right)\right| .
$$

In view of (5.4) and (5.6), we have

$$
\begin{aligned}
\left\|\boldsymbol{\Pi}_{h} \mathbf{u}-\mathbf{u}_{h}^{t}\right\|_{L^{\infty}} & \leq 2\left|\left(\boldsymbol{\Pi}_{h} \mathbf{u}-\mathbf{u}_{h}^{t}, \boldsymbol{\delta}_{1}\right)\right| \\
& =2\left|a_{\mathbf{u}}\left(\mathbf{G}_{1}^{h}, \boldsymbol{\Pi}_{h} \mathbf{u}-\mathbf{u}_{h}^{t}\right)+a_{p}\left(\boldsymbol{\Pi}_{h} \mathbf{u}-\mathbf{u}_{h}^{t}, \zeta_{1}^{h}\right)\right|
\end{aligned}
$$


By (2.20) together with (5.5) and (5.6), the above expressions can be expanded as

$$
\begin{aligned}
& \left(\boldsymbol{\Pi}_{h} \mathbf{u}-\mathbf{u}_{h}^{t}, \boldsymbol{\delta}_{1}\right) \\
& =a_{\mathbf{u}}\left(\mathbf{G}_{1}^{h}, \boldsymbol{\Pi}_{h} \mathbf{u}-\mathbf{u}_{h}^{t}\right)+a_{p}\left(\boldsymbol{\Pi}_{h} \mathbf{u}-\mathbf{u}_{h}^{t}, \zeta_{1}^{h}\right) \\
& =a_{\mathbf{u}}\left(\mathbf{G}_{1}^{h}-\mathbf{G}_{1}, \boldsymbol{\Pi}_{h} \mathbf{u}-\mathbf{u}\right)+a_{p}\left(\boldsymbol{\Pi}_{h} \mathbf{u}-\mathbf{u}, \zeta_{1}^{h}-\zeta_{1}\right)+a_{p}\left(p-\mathcal{Q}_{h} p, \mathbf{G}_{1}-\mathbf{G}_{1}^{h}\right) \\
& +\lambda^{-1}\left(p-\mathcal{Q}_{h} p, \zeta_{1}^{h}-\zeta_{1}\right)+a_{\mathbf{u}}\left(\mathbf{G}_{1}, \boldsymbol{\Pi}_{h} \mathbf{u}-\mathbf{u}\right)+a_{p}\left(\mathbf{\Pi}_{h} \mathbf{u}-\mathbf{u}, \zeta_{1}\right) \\
& +t\left(p-p_{h}^{t}, \operatorname{div}\left(\operatorname{adj} \boldsymbol{\nabla}\left(\mathbf{u}-\mathbf{u}_{h}^{t}\right) \mathbf{G}_{1}^{h}\right)\right)+t\left(\operatorname{det} \boldsymbol{\nabla}\left(\mathbf{u}-\mathbf{u}_{h}^{t}\right), \zeta_{1}^{h}\right) \\
& =: I_{1}+\cdots+I_{8} .
\end{aligned}
$$

Now we estimate $I_{1}$ to $I_{8}$. An application of Hölder's inequality yields a bound of $I_{1}$ to $I_{4}$ :

$$
\begin{aligned}
\left|I_{1}\right|+\cdots+\left|I_{4}\right| & \leq C\left(\left\|\boldsymbol{\nabla}\left(\mathbf{u}-\mathbf{\Pi}_{h} \mathbf{u}\right)\right\|_{L^{\infty}}+\left\|p-\mathcal{Q}_{h} p\right\|_{L^{\infty}}\right) \\
& \times\left(\left\|\boldsymbol{\nabla}\left(\mathbf{G}_{1}-\mathbf{G}_{1}^{h}\right)\right\|_{L^{1}}+\left\|\zeta_{1}-\zeta_{1}^{h}\right\|_{L^{1}}\right) .
\end{aligned}
$$

Recalling (5.5) we have

$$
\begin{aligned}
\left|I_{5}+I_{6}\right| & =\left|a_{\mathbf{u}}\left(\mathbf{G}_{1}, \boldsymbol{\Pi}_{h} \mathbf{u}-\mathbf{u}\right)+a_{p}\left(\boldsymbol{\Pi}_{h} \mathbf{u}-\mathbf{u}, \zeta_{1}\right)\right| \\
& =\left|\left(\boldsymbol{\delta}_{1}, \boldsymbol{\Pi}_{h} \mathbf{u}-\mathbf{u}\right)\right| \leq\left\|\boldsymbol{\delta}_{1}\right\|_{L^{1}}\left\|\boldsymbol{\Pi}_{h} \mathbf{u}-\mathbf{u}\right\|_{L^{\infty}}=\left\|\boldsymbol{\Pi}_{h} \mathbf{u}-\mathbf{u}\right\|_{L^{\infty}} .
\end{aligned}
$$

$I_{7}$ and $I_{8}$ are dealt with by using Hölder's inequality as

$$
\left|I_{7}\right| \leq t\left\|p-p_{h}^{t}\right\|_{L^{\infty}}\left\|\nabla\left(\mathbf{u}-\mathbf{u}_{h}^{t}\right)\right\|_{L^{\infty}}\left\|\nabla \mathbf{G}_{1}^{h}\right\|_{L^{1}}
$$

and

$$
\left|I_{8}\right| \leq C t\left\|\nabla\left(\mathbf{u}-\mathbf{u}_{h}\right)\right\|_{L^{\infty}}^{2}\left\|\zeta_{1}^{h}\right\|_{L^{1}}
$$

From (3.10) and Hölder's inequality we obtain

$$
\left\|\nabla\left(\mathbf{G}_{1}-\mathbf{G}_{1}^{h}\right)\right\|_{L^{1}} \leq C|\ln h|^{1 / 2}\left\|\nabla\left(\mathbf{G}_{1}-\mathbf{G}_{1}^{h}\right)\right\|_{\sigma_{1}^{2}},
$$

as well as similar inequalities for $\boldsymbol{\nabla} \mathbf{G}_{1}^{h}, \zeta_{1}-\zeta_{1}^{h}$, and $\boldsymbol{\nabla} \zeta_{1}^{h}$. Summing up all the above estimates, we complete the proof of (5.7).

Similarly, we define another two families of regularized Green's functions and their discrete versions.

Lemma 5.2. Let $\left(\mathbf{G}_{2}, \zeta_{2}\right) \in \mathbf{X} \times M$ and the corresponding regularized Green's function $\left(\mathbf{G}_{2}^{h}, \zeta_{2}^{h}\right) \in \mathbf{X}_{h} \times M_{h}$ satisfy

$$
\begin{array}{ll}
\mathcal{A}\left(\mathbf{G}_{2}, \zeta_{2} ; \mathbf{v}, q\right)=\left(\mathcal{D} \boldsymbol{\delta}_{2}, \mathbf{v}\right), & \forall(\mathbf{v}, q) \in \mathbf{X} \times M, \\
\mathcal{A}\left(\mathbf{G}_{2}^{h}, \zeta_{2}^{h} ; \mathbf{v}, q\right)=\left(\mathcal{D} \boldsymbol{\delta}_{2}, \mathbf{v}\right), & \forall(\mathbf{v}, q) \in \mathbf{X}_{h} \times M_{h},
\end{array}
$$

respectively. Here $\mathcal{D} \boldsymbol{\delta}_{2}$ stands for any direction derivatives of either $\boldsymbol{\delta}_{2}=\left(\delta_{2}, 0\right)$ or $\boldsymbol{\delta}_{2}=\left(0, \delta_{2}\right)$. Then we have

$$
\begin{aligned}
\left\|\boldsymbol{\nabla}\left(\mathbf{u}-\mathbf{u}_{h}^{t}\right)\right\|_{L^{\infty}} \leq & 2\left\|\boldsymbol{\nabla}\left(\mathbf{u}-\boldsymbol{\Pi}_{h} \mathbf{u}\right)\right\|_{L^{\infty}} \\
& +C|\ln h|^{1 / 2}\left(\left\|\boldsymbol{\nabla}\left(\mathbf{u}-\mathbf{\Pi}_{h} \mathbf{u}\right)\right\|_{L^{\infty}}+\left\|p-\mathcal{Q}_{h} p\right\|_{L^{\infty}}\right) \\
& \times\left(\left\|\boldsymbol{\nabla}\left(\mathbf{G}_{2}-\mathbf{G}_{2}^{h}\right)\right\|_{\sigma_{2}^{2}}+\left\|\zeta_{2}-\zeta_{2}^{h}\right\|_{\sigma_{2}^{2}}\right) \\
& +C t|\ln h|^{1 / 2}\left\|\boldsymbol{\nabla}\left(\mathbf{u}-\mathbf{u}_{h}^{t}\right)\right\|_{L^{\infty}}^{2}\left\|\zeta_{2}^{h}\right\|_{\sigma_{2}^{2}} \\
& +C t|\ln h|^{1 / 2}\left\|\boldsymbol{\nabla}\left(\mathbf{u}-\mathbf{u}_{h}^{t}\right)\right\|_{L^{\infty}}\left\|p-p_{h}^{t}\right\|_{L^{\infty}}\left\|\nabla \mathbf{G}_{2}^{h}\right\|_{\sigma_{2}^{2}} .
\end{aligned}
$$


Proof. The proof of this lemma is almost the same as for Lemma 5.1. The only difference is that we choose $\mathbf{x}_{2}$ to maximize $\left|\nabla\left(\Pi_{h} \mathbf{u}-\mathbf{u}_{h}^{t}\right)\right|$. By using (5.4) again, we obtain

$$
\begin{aligned}
\left\|\boldsymbol{\nabla}\left(\boldsymbol{\Pi}_{h} \mathbf{u}-\mathbf{u}_{h}^{t}\right)\right\|_{L^{\infty}} & \leq 2\left|\left(\boldsymbol{\nabla}\left(\boldsymbol{\Pi}_{h} \mathbf{u}-\mathbf{u}_{h}^{t}\right), \boldsymbol{\delta}_{2}\right)\right|=2\left|\left(\boldsymbol{\Pi}_{h} \mathbf{u}-\mathbf{u}_{h}^{t}, \mathcal{D} \boldsymbol{\delta}_{2}\right)\right| \\
& =2\left|a_{\mathbf{u}}\left(\mathbf{G}_{2}^{h}, \boldsymbol{\Pi}_{h} \mathbf{u}-\mathbf{u}_{h}^{t}\right)+a_{p}\left(\boldsymbol{\Pi}_{h} \mathbf{u}-\mathbf{u}_{h}^{t}, \zeta_{2}^{h}\right)\right| .
\end{aligned}
$$

The rest of the proof proceeds like the proof of Lemma 5.1 .

As in the preceding lemmas, we get

Lemma 5.3. Let $\left(\mathbf{G}_{3}, \zeta_{3}\right) \in \mathbf{X} \times M$ and the corresponding regularized Green's function $\left(\mathbf{G}_{3}^{h}, \zeta_{3}^{h}\right) \in \mathbf{X}_{h} \times M_{h}$ satisfy

$$
\begin{array}{ll}
\mathcal{A}\left(\mathbf{G}_{3}, \zeta_{3} ; \mathbf{v}, q\right)=\left(\delta_{3}, q\right), & \forall(\mathbf{v}, q) \in \mathbf{X} \times M, \\
\mathcal{A}\left(\mathbf{G}_{3}^{h}, \zeta_{3}^{h} ; \mathbf{v}, q\right)=\left(\delta_{3}, q\right), & \forall(\mathbf{v}, q) \in \mathbf{X}_{h} \times M_{h},
\end{array}
$$

respectively. Then we have

$$
\begin{aligned}
\left\|p-p_{h}^{t}\right\|_{L^{\infty}} & \leq 2\left\|p-\mathcal{Q}_{h} p\right\|_{L^{\infty}}+C|\ln h|^{1 / 2}\left(\left\|\boldsymbol{\nabla}\left(\mathbf{u}-\boldsymbol{\Pi}_{h} \mathbf{u}\right)\right\|_{L^{\infty}}+\left\|p-\mathcal{Q}_{h} p\right\|_{L^{\infty}}\right) \\
& \times\left(\left\|\nabla\left(\mathbf{G}_{3}-\mathbf{G}_{3}^{h}\right)\right\|_{\sigma_{3}^{2}}+\left\|\zeta_{3}-\zeta_{3}^{h}\right\|_{\sigma_{3}^{2}}\right) \\
& +C t|\ln h|^{1 / 2}\left\|\boldsymbol{\nabla}\left(\mathbf{u}-\mathbf{u}_{h}^{t}\right)\right\|_{L^{\infty}}^{2}\left\|\zeta_{3}^{h}\right\|_{\sigma_{3}^{2}} \\
& +C t|\ln h|^{1 / 2}\left\|\boldsymbol{\nabla}\left(\mathbf{u}-\mathbf{u}_{h}^{t}\right)\right\|_{L^{\infty}}\left\|p-p_{h}^{t}\right\|_{L^{\infty}}\left\|\nabla \mathbf{G}_{3}^{h}\right\|_{\sigma_{3}^{2}} .
\end{aligned}
$$

When applying the three lemmas above, we need bounds of $\mathbf{G}_{i}, \mathbf{G}_{i}^{h}, \zeta_{i}, \zeta_{i}^{h}$, and

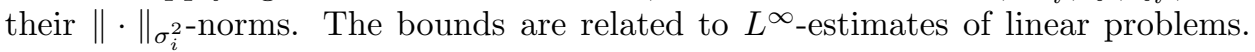
Therefore, the proofs are postponed to the appendix.

Lemma 5.4. There exists a constant $C$ which may depend on $\|\mathbf{u}\|_{\mathcal{C}^{2}(\bar{\Omega})}$ and $\|p\|_{\mathcal{C}^{1}(\bar{\Omega})}$ such that

$$
\begin{aligned}
&\left\|\mathcal{D}^{2} \mathbf{G}_{1}\right\|_{\sigma_{i}^{2}}+\left\|\boldsymbol{\nabla} \zeta_{1}\right\|_{\sigma_{i}^{2}} \leq \begin{cases}C|\ln h|^{1 / 2}, & i=1, \\
C h^{-1}, & i=2,3,\end{cases} \\
&\left\|\nabla \mathbf{G}_{i}\right\|_{\sigma_{i}^{2}}+\left\|\zeta_{i}\right\|_{\sigma_{i}^{2}} \leq C|\ln h|^{1 / 2}, i=1,2,3 .
\end{aligned}
$$

Theorem 5.5. Let $\mathbf{G}_{i}$ and $\mathbf{G}_{i}^{h}$ be defined as before. If $h$ is small enough,

$$
\left\|\boldsymbol{\nabla}\left(\mathbf{G}_{i}-\mathbf{G}_{i}^{h}\right)\right\|_{\sigma_{1}^{2}}+\left\|\zeta_{i}-\zeta_{i}^{h}\right\|_{\sigma_{1}^{2}} \leq \begin{cases}C h|\ln h|^{1 / 2}, & \text { for } i=1, \\ C, & \text { for } i=2,3 .\end{cases}
$$

\section{Proof of the main Result}

In this section, we present the proof of Theorem 2.5. As preparation, we first prove Lemma 2.10 and Lemma 2.11

Proof of Lemma 2.10, Expressions with the discrete Green's functions will be estimated by applying the triangle inequality to $\boldsymbol{\nabla} \mathbf{G}_{i}^{h}=\boldsymbol{\nabla G}_{i}+\left(\boldsymbol{\nabla} \mathbf{G}_{i}^{h}-\boldsymbol{\nabla} \mathbf{G}_{i}\right)$ and $\zeta_{i}^{h}=\zeta_{i}+\left(\zeta_{i}^{h}-\zeta_{i}\right)$. Inserting (5.16) into Lemma 5.2 and Lemma 5.3, respectively, and recalling (5.15), we obtain the a priori estimates

$$
\begin{aligned}
\left\|\boldsymbol{\nabla}\left(\mathbf{u}-\mathbf{u}_{h}^{t}\right)\right\|_{L^{\infty}}+\left\|p-p_{h}^{t}\right\|_{L^{\infty}} & \leq C h|\ln h|^{1 / 2}+C t|\ln h|\left\|\nabla\left(\mathbf{u}-\mathbf{u}_{h}^{t}\right)\right\|_{L^{\infty}} \\
& \times\left(\left\|\nabla\left(\mathbf{u}-\mathbf{u}_{h}^{t}\right)\right\|_{L^{\infty}}+\left\|p-p_{h}^{t}\right\|_{L^{\infty}}\right) .
\end{aligned}
$$


Similarly, inserting (5.16) into Lemma [5.1, we have

$$
\begin{aligned}
\left\|\mathbf{u}-\mathbf{u}_{h}^{t}\right\|_{L^{\infty}} & \leq C h^{2}|\ln h| \\
& +C t|\ln h|\left\|\boldsymbol{\nabla}\left(\mathbf{u}-\mathbf{u}_{h}^{t}\right)\right\|_{L^{\infty}}\left(\left\|\boldsymbol{\nabla}\left(\mathbf{u}-\mathbf{u}_{h}^{t}\right)\right\|_{L^{\infty}}+\left\|p-p_{h}^{t}\right\|_{L^{\infty}}\right) .
\end{aligned}
$$

Without loss of generality we may assume that $C \geq 1$ and $h \leq e^{-1}$. Setting

$$
A:=\left\|\nabla\left(\mathbf{u}-\mathbf{u}_{h}^{t}\right)\right\|_{L^{\infty}}+\left\|p-p_{h}^{t}\right\|_{L^{\infty}}, \quad B:=\left\|\mathbf{u}-\mathbf{u}_{h}^{t}\right\|_{L^{\infty}},
$$

the two inequalities above may be rewritten as

$$
\begin{aligned}
& A \leq C h|\ln h|^{1 / 2}+C|\ln h| A^{2}, \\
& B \leq C h^{2}|\ln h|+C|\ln h| A^{2} .
\end{aligned}
$$

Referring to the norm $\|\cdot\|_{h}$ that has been defined in (2.17), we assume that

$$
\left\|\mathbf{u}-\mathbf{u}_{h}^{t}, p-p_{h}^{t}\right\|_{h}=h|\ln h|^{3 / 2} A+B \leq \gamma:=\frac{1}{2 C} h|\ln h|^{1 / 2} .
$$

From the inequality for $A$ and the restriction (6.3) we conclude that

$$
\begin{aligned}
A & \leq C h|\ln h|^{1 / 2}+C|\ln h| A h^{-1}|\ln h|^{-3 / 2} \gamma \\
& \leq C h|\ln h|^{1 / 2}+\frac{1}{2} A \leq 2 C h|\ln h|^{1 / 2} .
\end{aligned}
$$

Combining the inequality for $B$ with (6.4), we obtain

$$
B \leq C h^{2}|\ln h|+C|\ln h| 4 C^{2} h^{2}|\ln h| \leq 5 C^{3} h^{2}|\ln h|^{2} .
$$

Finally we assume that

$$
h \leq h_{1}, \quad \text { where } \quad h_{1}\left|\ln h_{1}\right|^{3 / 2} \leq \frac{1}{28 C^{4}} .
$$

Hence,

$$
\begin{aligned}
h|\ln h|^{3 / 2} A+B & \leq 2 C h^{2}|\ln h|^{2}+5 C^{3} h^{2}|\ln h|^{2} \leq 7 C^{3} h^{2}|\ln h|^{2} \\
& \leq \frac{1}{4 C} h|\ln h|^{1 / 2} \leq \frac{1}{2} \gamma .
\end{aligned}
$$

After setting $\mathcal{K}_{1}:=\max \left(2 C, 7 C^{3}\right)=7 C^{3}$, the proof of the first assertion is complete. Note that for $t=0$,

$$
\left\|\mathbf{u}-\mathbf{u}_{h}^{0}, p-p_{h}^{0}\right\|_{h} \leq 2 C h^{2}|\ln h|^{2}<\mathcal{K}_{1} h^{2}|\ln h|^{2} .
$$

Thus the second assertion follows.

Proof of Lemma[2.11] Let $(\overline{\mathbf{u}}, \bar{p}) \in U_{\mathcal{K}_{1} h^{2}|\ln h|^{2}}$. We want to show that the problem

$$
\mathcal{D H}(t,(\overline{\mathbf{u}}, \bar{p}))\left(\left(\mathbf{w}_{h}, r_{h}\right),(\phi, s)\right)=0, \quad \forall(\phi, s) \in \mathbf{X}_{h} \times M_{h}
$$

has only the trivial solution $\left(\mathbf{w}_{h}, r_{h}\right)=0$ whenever $h$ is sufficiently small. Note that for any $(\phi, s) \in \mathbf{X}_{h} \times M_{h}$,

$$
\mathcal{D H}(t,(\overline{\mathbf{u}}, \bar{p}))\left(\left(\mathbf{w}_{h}, r_{h}\right),(\phi, s)\right)=\mathcal{A}\left(\mathbf{w}_{h}, r_{h} ; \phi, s\right)+t M\left(\mathbf{w}_{h}, r_{h} ; \phi, s\right),
$$

where $M\left(\mathbf{w}_{h}, r_{h} ; \boldsymbol{\phi}, s\right)$ is defined as

$$
\begin{aligned}
M\left(\mathbf{w}_{h}, r_{h} ; \boldsymbol{\phi}, s\right):= & \left(\bar{p}-p, \operatorname{div}\left(\operatorname{adj} \boldsymbol{\nabla} \mathbf{w}_{h} \phi\right)\right)+\left(r_{h}, \operatorname{div}(\operatorname{adj} \boldsymbol{\nabla}(\mathbf{u}-\overline{\mathbf{u}}) \boldsymbol{\phi})\right) \\
& +\left(s, \operatorname{div}\left(\operatorname{adj} \boldsymbol{\nabla}(\mathbf{u}-\overline{\mathbf{u}}) \mathbf{w}_{h}\right)\right) .
\end{aligned}
$$


By Theorem A.3 we have sufficiently small $h$ :

$$
\begin{aligned}
& \sup _{(\boldsymbol{\phi}, s) \in \mathbf{X}_{h} \times M_{h}} \frac{\mathcal{D H}(t,(\overline{\mathbf{u}}, \bar{p}))\left(\left(\mathbf{w}_{h}, r_{h}\right),(\boldsymbol{\phi}, s)\right)}{\|\phi\|_{1}+\|s\|_{0}} \\
& \geq C\left(\left\|\mathbf{w}_{h}\right\|_{1}+\left\|r_{h}\right\|_{0}\right)-C t\left(\|\boldsymbol{\nabla}(\mathbf{u}-\overline{\mathbf{u}})\|_{L^{\infty}}+\|p-\bar{p}\|_{L^{\infty}}\right)\left(\left\|\mathbf{w}_{h}\right\|_{1}+\left\|r_{h}\right\|_{0}\right) \\
& \geq C\left(\left\|\mathbf{w}_{h}\right\|_{1}+\left\|r_{h}\right\|_{0}\right) .
\end{aligned}
$$

This together with (6.7) leads to $\left(\mathbf{w}_{h}, r_{h}\right)=0$, and the proof is complete.

Now we are ready to prove the main theorem.

Proof of Theorem 2.5. We will show that for sufficiently small $h$ the set $\Xi_{h}\left(\mathcal{K}_{1}\right)$ is nonvoid, open and closed with respect to the interval $[0,1]$. Hence, it coincides with $[0,1]$.

Obviously, $0 \in \Xi_{h}\left(\mathcal{K}_{1}\right)$ by virtue of the last assertion of Lemma 2.10

We claim that $\Xi_{h}\left(\mathcal{K}_{1}\right)$ is open in $[0,1]$. If $\tau \in \Xi_{h}\left(\mathcal{K}_{1}\right)$, we have $\mathcal{H}\left(\tau,\left(\mathbf{u}_{h}^{\tau}, p_{h}^{\tau}\right)\right)=0$ and $\mathcal{D H}\left(\tau,\left(\mathbf{u}_{h}^{\tau}, p_{h}^{\tau}\right)\right)$ is regular by Lemma 2.11. By the implicit function theorem [14. Theorem A] there exists a neighborhood $\mathcal{O}_{1}(\tau) \subset[0,1]$ such that for all $t \in$ $\mathcal{O}_{1}(\tau)$

(1) the nonlinear equation $\mathcal{H}(t,(\mathbf{u}, p))=0$ has a solution $\left(\mathbf{u}_{h}^{t}, p_{h}^{t}\right)$;

(2) $\left(\mathbf{u}_{h}^{t}, p_{h}^{t}\right)$ is continuous in $t$ with respect to the norm $\|\cdot\|_{h}$.

Since $\left(\mathbf{u}_{h}^{\tau}, p_{h}^{\tau}\right) \in U_{\mathcal{K}_{1} h^{2}|\ln h|^{2}}$, by (2) there exists a neighborhood $\mathcal{O}_{2}(\tau)$ of $\tau$ such that for all $t \in \mathcal{O}_{2}(\tau)$, we have

$$
\left(\mathbf{u}_{h}^{t}, p_{h}^{t}\right) \in U_{2 \mathcal{K}_{1} h^{2}|\ln h|^{2}} .
$$

An application of Lemma 2.10 yields

$$
\left(\mathbf{u}_{h}^{t}, p_{h}^{t}\right) \in U_{\mathcal{K}_{1} h^{2}|\ln h|^{2}} .
$$

Thus $t \in \Xi_{h}\left(\mathcal{K}_{1}\right)$ for all $t \in \mathcal{O}_{1}(\tau) \cap \mathcal{O}_{2}(\tau)$, and $\Xi_{h}\left(\mathcal{K}_{1}\right)$ is open.

The set $\Xi_{h}\left(\mathcal{K}_{1}\right)$ also is closed. Indeed, let $t_{k} \in \Xi_{h}\left(\mathcal{K}_{1}\right)$ be parameters tending to $\tau \in[0,1]$ and let $\left(\mathbf{u}_{h}^{t_{k}}, p_{h}^{t_{k}}\right)$ be the corresponding solutions of Problem [2.9. By virtue of the compactness of $U_{\mathcal{K}_{1} h^{2}|\ln h|^{2}} \cap\left(\mathbf{X}_{h} \times M_{h}\right)$, a subsequence converges to some $\left(\mathbf{u}_{h}^{\tau}, p_{h}^{\tau}\right) \in U_{\mathcal{K}_{1} h^{2}|\ln h|^{2}} \cap\left(\mathbf{X}_{h} \times M_{h}\right)$. Since $\mathcal{H}\left(t_{k},\left(\mathbf{u}_{h}^{t_{k}}, p_{h}^{t_{k}}\right)\right)=0$, it follows from the continuity that $\mathcal{H}\left(\tau,\left(\mathbf{u}_{h}^{\tau}, p_{h}^{\tau}\right)\right)=0$. Applying Lemma 2.10 once more, we see that the solutions $\left(\mathbf{u}_{h}^{\tau}, p_{h}^{\tau}\right)$ satisfy the strict inequality in (2.21). This proves $\tau \in \Xi_{h}\left(\mathcal{K}_{1}\right)$, and $\Xi_{h}\left(\mathcal{K}_{1}\right)$ is closed.

Consequently, we have $\Xi_{h}\left(\mathcal{K}_{1}\right)=[0,1]$ which proves Theorem 2.5 .

Remark 6.1. Until now we have disregarded the orientation preserving condition. This is justified by the fact that the finite element solution $\mathbf{u}_{h}$ is orientation preserving, i.e.,

$$
\operatorname{det}\left(\operatorname{Id}+\boldsymbol{\nabla} \mathbf{u}_{h}\right)>0 \text { in } \Omega
$$

for sufficiently small $h$, provided that the solution of the continuous problem $\mathbf{u}$ has this property.

Indeed, since $\mathbf{u} \in \mathcal{C}^{1}(\Omega)$, the determinant is continuous and bounded from below. It follows that

$$
\begin{aligned}
\operatorname{det}\left(\operatorname{Id}+\boldsymbol{\nabla} \mathbf{u}_{h}\right) & =\operatorname{det}(\operatorname{Id}+\boldsymbol{\nabla u})+\operatorname{Cof}(\operatorname{Id}+\boldsymbol{\nabla u}): \boldsymbol{\nabla}\left(\mathbf{u}_{h}-\mathbf{u}\right)+\operatorname{det} \boldsymbol{\nabla}\left(\mathbf{u}_{h}-\mathbf{u}\right) \\
& \geq \operatorname{det}(\operatorname{Id}+\boldsymbol{\nabla u})-C h|\ln h|^{1 / 2}-C h^{2}|\ln h| \geq \frac{1}{2} \operatorname{det}(\operatorname{Id}+\boldsymbol{\nabla u})>0
\end{aligned}
$$


if $h$ is small, and the deformation is orientation preserving.

\section{Appendix A. Discretization error of Green's functions}

The aim of this appendix is the proof of Theorem [5.5, i.e., we are concerned with the estimates of $\mathbf{G}_{i}-\mathbf{G}_{i}^{h}$ and $\zeta_{i}-\zeta_{i}^{h}$ in the weighted norms. The Green's functions are defined by linear problems and, in essence, we have to perform those steps which make the crucial part of $L^{\infty}$-error estimates for the linear variational Problem 2.3.

As already mentioned, Problem [2.3 has some special features. It is a saddle point problem with a penalty term. Fortunately that term belongs to a bounded operator; cf. [5]. Moreover there is only a Gårding inequality for $a_{u}(.,$.$) and no$ coercivity. Based on the observation in [34], we will restrict ourselves to small $h$.

The formulation of $\mathbf{G}_{i}^{h}$ and $\zeta_{i}^{h}$ can be embedded into Problem 2.3. Since the following considerations are independent of $i$, the subscript $i$ is dropped whenever possible.

The stability of Problem 2.3 is now established via the discrete $L B B$ condition, a Gärding inequality, and a compactness argument. To this end another norm will be used. For any $(\mathbf{v}, q) \in \mathbf{X}_{h} \times M_{h}$, we define

$$
\|\mathbf{v}, q\|^{2}:=\|\mathbf{v}\|_{1}^{2}+\|q\|_{0}^{2} .
$$

Lemma A.1. There exists a constant $C>0$ such that for sufficiently small $h$,

$$
\sup _{\mathbf{v} \in \mathbf{X}_{h}} \frac{a_{p}(\mathbf{v}, q)}{\|\mathbf{v}\|_{1}} \geq C\|q\|_{0}, \quad \forall q \in M_{h} .
$$

Proof. The proof of this discrete $L B B$ inequality is just a combination of the $L B B$ inequality (2.14) and the interpolant $\Pi_{h}$ (4.2). The latter serves as a Fortin-like operator [17, 7, p. 136]. For a matrix $\mathbf{A}, \operatorname{adj}_{h} \mathbf{A}$ is defined by

$$
\left(\operatorname{adj}_{h} \mathbf{A}\right)_{i j}:=\left(\mathcal{Q}_{h} \operatorname{adj} \mathbf{A}\right)_{i j}=\mathcal{Q}_{h}(\operatorname{adj} A)_{i j},
$$

and $\operatorname{Cof}_{h} \mathbf{A}$ is defined in the same manner.

Given $q \in M_{h}$, by the $L B B$ inequality (2.14), there exists $\mathbf{v} \in \mathbf{X}$ such that

$$
a_{p}(\mathbf{v}, q)=\|q\|_{0}^{2} \quad \text { and } \quad\|\mathbf{v}\|_{1} \leq C_{b}\|q\|_{0} .
$$

By definition of $a_{p}$ we have

$$
\begin{aligned}
a_{p}\left(\boldsymbol{\Pi}_{h} \mathbf{v}, q\right)=a_{p}(\mathbf{v}, q) & +\left(q, \operatorname{div}\left(\operatorname{adj}_{h}(\operatorname{Id}+\boldsymbol{\nabla} \mathbf{u})\left(\boldsymbol{\Pi}_{h} \mathbf{v}-\mathbf{v}\right)\right)\right) \\
& +\left(q, \operatorname{div}\left(\left(\operatorname{adj}-\operatorname{adj}_{h}\right)(\operatorname{Id}+\boldsymbol{\nabla} \mathbf{u})\left(\boldsymbol{\Pi}_{h} \mathbf{v}-\mathbf{v}\right)\right)\right) .
\end{aligned}
$$

Here, we understand

$$
\left(q, \operatorname{div}\left(\operatorname{adj}_{h}(\operatorname{Id}+\nabla \mathbf{u}) \boldsymbol{\Pi}_{h} \mathbf{v}\right)\right)=\sum_{K \in \mathcal{T}_{2 h}}\left(q, \operatorname{div}\left(\operatorname{adj}_{h}(\operatorname{Id}+\nabla \mathbf{u}) \boldsymbol{\Pi}_{h} \mathbf{v}\right)\right)_{K}
$$

This is justified by the fact that $\operatorname{adj}_{h}(\mathrm{Id}+\boldsymbol{\nabla u})$ is a piecewise constant matrix, and due to (4.1) 3 no terms with inner boundaries arise when Green's formula is applied. From (4.3) we conclude that the second term on the right-hand side of (A.3) vanishes and using the Piola identity (2.6),

$$
\begin{aligned}
a_{p}\left(\boldsymbol{\Pi}_{h} \mathbf{v}, q\right) & =a_{p}(q, \mathbf{v})+\left(q,\left(\operatorname{Cof}-\operatorname{Cof}_{h}\right)(\mathrm{Id}+\boldsymbol{\nabla} \mathbf{u}): \nabla\left(\boldsymbol{\Pi}_{h} \mathbf{v}-\mathbf{v}\right)\right) \\
& \geq\|q\|_{0}^{2}-C_{1} h\|q\|_{0}\left\|\boldsymbol{\nabla}\left(\mathbf{v}-\boldsymbol{\Pi}_{h} \mathbf{v}\right)\right\|_{0}
\end{aligned}
$$


From (4.7) and (A.2) it follows that

$$
\left\|\boldsymbol{\nabla}\left(\mathbf{v}-\boldsymbol{\Pi}_{h} \mathbf{v}\right)\right\|_{0} \leq\|\boldsymbol{\nabla} \mathbf{v}\|_{0}+\left\|\boldsymbol{\nabla} \boldsymbol{\Pi}_{h} \mathbf{v}\right\|_{0} \leq C\|\nabla \mathbf{v}\|_{0} \leq C\|q\|_{0} .
$$

A combination of the above two inequalities gives (A.1) for sufficiently small $h$.

Lemma A.2. There exist constants $C_{1}$ and $C_{2}$ such that for sufficiently small $h$,

$$
\sup _{(\mathbf{w}, r) \in \mathbf{X}_{h} \times M_{h}} \frac{\mathcal{A}(\mathbf{w}, r ; \mathbf{v}, q)}{\|\mathbf{w}, r\|} \geq C_{1}\|\mathbf{v}, q\|-C_{2}\|\mathbf{v}\|_{0}, \quad \forall(\mathbf{v}, q) \in \mathbf{X}_{h} \times M_{h} .
$$

Proof. In view of the discrete $L B B$ inequality (A.1), there exists $C_{b}>0$ such that

$$
a_{p}\left(\mathbf{v}_{1}, q\right)=\|q\|_{0}^{2} \quad \text { and } \quad\left\|\mathbf{v}_{1}\right\|_{1} \leq C_{b}\|q\|_{1} .
$$

Set $C_{s}:=1+\|p\|_{\mathcal{C}^{0}(\bar{\Omega})}$, and let $C_{p}$ be the constant in the Poincaré's inequality, i.e., $\|\mathbf{v}\|_{1} \leq C_{p}\|\boldsymbol{\nabla} \mathbf{v}\|_{0}$. From Theorem 2.4 it follows that for any $\alpha>0$

$$
\begin{aligned}
\mathcal{A}\left(\mathbf{v}+\alpha \mathbf{v}_{1},-q ; \mathbf{v}, q\right)= & \mathcal{A}(\mathbf{v},-q ; \mathbf{v}, q)+\alpha \mathcal{A}\left(\mathbf{v}_{1}, 0 ; \mathbf{v}, q\right) \\
= & a_{u}(\mathbf{v}, \mathbf{v})+\lambda^{-1}\|q\|_{0}^{2}+\alpha a_{\mathbf{u}}\left(\mathbf{v}_{1}, \mathbf{v}\right)+\alpha a_{p}\left(\mathbf{v}_{1}, q\right) \\
\geq & 1 / 2\|\nabla \mathbf{v}\|_{0}^{2}-1 / 2\|p\|_{\mathcal{C}^{1}(\bar{\Omega})}^{2}\|\mathbf{v}\|_{0}^{2}+\lambda^{-1}\|q\|_{0}^{2} \\
& +\alpha / 2\|q\|_{0}^{2}-\alpha C_{b}^{2} C_{s}^{2} / 2\|\mathbf{\nabla} \mathbf{v}\|_{0}^{2} \\
\geq & \frac{1-\alpha C_{b} C_{s}^{2}}{2 C_{p}}\|\mathbf{v}\|_{1}^{2}+\frac{\alpha}{2}\|q\|_{0}^{2}+\lambda^{-1}\|q\|_{0}^{2}-\frac{1}{2}\|p\|_{\mathcal{C}^{1}(\bar{\Omega})}^{2}\|\mathbf{v}\|_{0}^{2} .
\end{aligned}
$$

Next we choose $\alpha:=C_{p} /\left(1+C_{p} C_{b}^{2} C_{s}^{2}\right)$. Since $\frac{1}{2}\left(1-\alpha C_{b} C_{s}^{2}\right) C_{p}=\alpha / 2$, it follows that

$$
\begin{aligned}
\mathcal{A}\left(\mathbf{v}+\alpha \mathbf{v}_{1},-q ; \mathbf{v}, q\right) & \geq \alpha / 2\|\mathbf{v}, q\|^{2}-1 / 2\|p\|_{\mathcal{C}^{1}(\bar{\Omega})}^{2}\|\mathbf{v}\|_{0}^{2} \\
& \geq\left(\alpha / 2\|\mathbf{v}, q\|-1 / 2\|p\|_{\mathcal{C}^{1}(\bar{\Omega})}^{2}\|\mathbf{v}\|_{0}\right)\|\mathbf{v}, q\| .
\end{aligned}
$$

By construction,

$$
\left\|\mathbf{v}+\alpha \mathbf{v}_{1},-q\right\| \leq \max \left(\sqrt{2}, 1+\sqrt{2} \alpha C_{b}\right)\|\mathbf{v}, q\|=: C_{3}\|\mathbf{v}, q\| .
$$

A combination of the last two inequalities yields (A.4) with $C_{1}:=\alpha /\left(2 C_{3}\right)$ and $C_{2}:=\|p\|_{\mathcal{C}^{1}(\bar{\Omega})}^{2} /\left(2 C_{3}\right)$.

Theorem A.3. If $h$ is sufficiently small, then there exists a constant $C$ such that

$$
\sup _{(\mathbf{w}, r) \in \mathbf{X}_{h} \times M_{h}} \frac{\mathcal{A}(\mathbf{w}, r ; \mathbf{v}, q)}{\|\mathbf{w}, r\|} \geq C\|\mathbf{v}, q\| \quad \forall(\mathbf{v}, q) \in \mathbf{X}_{h} \times M_{h} .
$$

Proof. We use a contradiction argument. Suppose that A.5 does not hold. Then there exists a sequence $\left\{\mathbf{v}_{n}, q_{n}\right\} \in \mathbf{X}_{h_{n}} \times M_{h_{n}}$ such that $h_{n}<1 / n,\left\|\mathbf{v}_{n}, q_{n}\right\|=1$, and

$$
\left|\mathcal{A}\left(\mathbf{w}, r ; \mathbf{v}_{n}, q_{n}\right)\right| \leq \frac{1}{n}\|\mathbf{w}, r\|, \quad \text { for all }(\mathbf{w}, r) \in \mathbf{X}_{h_{n}} \times M_{h_{n}} .
$$

So there exists a subsequence of $\left\{\mathbf{v}_{n}, q_{n}\right\}_{n=1}^{\infty}$ that will be denoted by $\left\{\mathbf{v}_{n}, q_{n}\right\}$ again, and $(\overline{\mathbf{v}}, \bar{q}) \in \mathbf{X} \times M$ such that

$$
\begin{array}{ll}
\mathbf{v}_{n} \rightarrow \overline{\mathbf{v}} & \text { weakly in } \mathbf{H}_{0}^{1}(\Omega), \mathbf{v}_{n} \rightarrow \overline{\mathbf{v}} \text { strongly in } \mathbf{L}^{2}(\Omega), \\
q_{n} \rightarrow \bar{q} & \text { weakly in } L^{2}(\Omega) .
\end{array}
$$


From the discrete Gårding inequality (A.4) and the assumption A.6), we obtain for large $n$ the inequality $C_{1}\left\|\mathbf{v}_{n}, q_{n}\right\|-C_{2}\left\|\mathbf{v}_{n}\right\|_{0} \leq \frac{1}{2} C_{1}\left\|\mathbf{v}_{n}, q_{n}\right\|$ which implies

$$
\left\|\mathbf{v}_{n}\right\|_{0} \geq C_{1} /\left(2 C_{2}\right)>0 \text {. }
$$

Hence, $\|\overline{\mathbf{v}}\|_{0} \geq C_{1} /\left(2 C_{2}\right)$ and $(\overline{\mathbf{v}}, \bar{q}) \neq 0$. By Assumption $\mathbf{R}$, there exists $(\mathbf{w}, r) \in$ $\mathbf{X} \times M$ with $\|\mathbf{w}, r\|=1$ such that

$$
\mathcal{A}(\mathbf{w}, r ; \overline{\mathbf{v}}, \bar{q}) \geq C_{4}>0 .
$$

The weak convergence of $\mathbf{v}_{n}$ and $q_{n}$ together with (A.7) implies that for sufficiently large $n$, it holds that

$$
\mathcal{A}\left(\mathbf{w}, r ; \mathbf{v}_{n}, q_{n}\right) \geq C_{4} / 2 .
$$

The density of the finite element spaces guarantees that there exists some $\left(\mathbf{w}_{n}, r_{n}\right) \in$ $\mathbf{X}_{h_{n}} \times M_{h_{n}}$ such that $\left\|\mathbf{w}-\mathbf{w}_{n}, r-r_{n}\right\| \leq C_{4} /(4\|\mathcal{L}\|)$, where $\|\mathcal{L}\|$ denotes the usual operator norm of $\mathcal{L}$. These estimates imply that for sufficiently large $n$, it holds that

$$
\begin{aligned}
\mathcal{A}\left(\mathbf{w}_{n}, r_{n} ; \mathbf{v}_{n}, q_{n}\right) & =\mathcal{A}\left(\mathbf{w}, r ; \mathbf{v}_{n}, q_{n}\right)-\mathcal{A}\left(\mathbf{w}-\mathbf{w}_{n}, r-r_{n} ; \mathbf{v}_{n}, q_{n}\right) \\
& \geq C_{4} / 2-\left\|\mathbf{w}-\mathbf{w}_{n}, r-r_{n}\right\|\|\mathcal{L}\| \\
& \geq C_{4} / 4 .
\end{aligned}
$$

This obviously contradicts (A.6), and the proof is complete.

Now we turn to the Green's functions. By construction, $\left\|\delta_{i}\right\|_{0} \leq C h^{-1},\left\|\nabla \delta_{i}\right\|_{0} \leq$ $\mathrm{Ch}^{-2}$, and as a simple corollary of Theorem 3.1, we have

Lemma A.4. There exists a constant $C$ which may depend on $\|\mathbf{u}\|_{\mathcal{C}^{2}(\bar{\Omega})}$ and $\|p\|_{\mathcal{C}^{1}(\bar{\Omega})}$ such that

$$
\left\|\mathcal{D}^{2} \mathbf{G}_{i}\right\|_{0}+\left\|\nabla \zeta_{i}\right\|_{0} \leq \begin{cases}C h^{-1}, & \text { for } i=1, \\ C h^{-2}, & \text { for } i=2,3 .\end{cases}
$$

Having Theorem A.3 we obtain the following error bounds by a standard scaling argument (see [7, Lemma III.3.7]) and the duality technique of Aubin-Nitsche.

Lemma A.5. If $h$ is sufficiently small, then we have

$$
\begin{aligned}
\left\|\boldsymbol{\nabla}\left(\mathbf{G}-\mathbf{G}^{h}\right)\right\|_{0}+\left\|\zeta-\zeta^{h}\right\|_{0} & \leq C h\left(\left\|\mathcal{D}^{2} \mathbf{G}\right\|_{0}+\|\boldsymbol{\nabla} \zeta\|_{0}\right), \\
\left\|\mathbf{G}-\mathbf{G}^{h}\right\|_{0}+\left\|\zeta-\zeta^{h}\right\|_{-1} & \leq C h^{2}\left(\left\|\mathcal{D}^{2} \mathbf{G}\right\|_{0}+\|\boldsymbol{\nabla} \zeta\|_{0}\right),
\end{aligned}
$$

where $C$ may depend on $\|\mathbf{u}\|_{\mathcal{C}^{2}(\bar{\Omega})}$ and $\|p\|_{\mathcal{C}^{1}(\bar{\Omega})}$.

Before estimating $\left\|\boldsymbol{\nabla}\left(\mathbf{G}-\mathbf{G}^{h}\right)\right\|_{\sigma^{2}}$ and $\left\|\zeta-\zeta^{h}\right\|_{\sigma^{2}}$, we cite an approximation result which will be frequently used in the subsequent analysis.

Lemma A.6. Let $K$ be as in (3.6), and let $\boldsymbol{\psi}:=\left(\mathbf{G}-\mathbf{G}^{h}\right) \sigma^{2}$. If $h$ is sufficiently small, then there exist constants $C_{K}>0$ and $C>0$ such that

$$
\begin{aligned}
\left\|\boldsymbol{\nabla}\left(\boldsymbol{\psi}-\boldsymbol{\Pi}_{h} \boldsymbol{\psi}\right)\right\|_{\sigma^{-2}} & \leq C_{K} h^{2}\left(\left\|\mathcal{D}^{2} \mathbf{G}\right\|_{0}+\|\boldsymbol{\nabla} \zeta\|_{0}\right) \\
& +C_{K} h\left(\left\|\mathcal{D}^{2} \mathbf{G}\right\|_{\sigma^{2}}+\|\boldsymbol{\nabla} \zeta\|_{\sigma^{2}}\right)+\frac{C}{K}\left\|\boldsymbol{\nabla}\left(\mathbf{G}-\mathbf{G}^{h}\right)\right\|_{\sigma^{2}} .
\end{aligned}
$$

This assertion follows from [16, Lemma 4.1] due to Theorem 4.1.

The following lemma is crucial for the estimate of $\left\|\boldsymbol{\nabla}\left(\mathbf{G}-\mathbf{G}^{h}\right)\right\|_{\sigma^{2}}$. It is similar to [16. Lemma 4.2]; the proof is, however, quite different. 
Lemma A.7. Let $\xi:=\left(\mathcal{Q}_{h} \zeta-\zeta^{h}\right) \sigma^{2}$ and $\psi:=\left(\mathbf{G}-\mathbf{G}^{h}\right) \sigma^{2}$. Then for any $\epsilon>0$, there exists a constant $C(\epsilon)$ which is independent of $h$ such that

$$
\begin{aligned}
& \left|a_{p}\left(\boldsymbol{\psi}, \zeta-\zeta^{h}\right)-\lambda^{-1}\left(\mathcal{Q}_{h} \zeta-\zeta^{h}, \xi\right)\right| \\
& \quad \leq \epsilon\left\|\boldsymbol{\nabla}\left(\mathbf{G}-\mathbf{G}^{h}\right)\right\|_{\sigma^{2}}^{2}+C(\epsilon) h^{4}\left(\left\|\mathcal{D}^{2} \mathbf{G}\right\|_{0}+\|\boldsymbol{\nabla} \zeta\|_{0}\right)^{2}+C(\epsilon) h^{2}\|\boldsymbol{\nabla} \zeta\|_{\sigma^{2}}^{2} .
\end{aligned}
$$

Proof. Integration by parts yields

$$
\begin{aligned}
a_{p}\left(\boldsymbol{\psi}, \zeta-\zeta^{h}\right) & =\left(\zeta-\zeta^{h}, \operatorname{Cof}(\operatorname{Id}+\nabla \mathbf{u}):\left(\mathbf{G}-\mathbf{G}^{h}\right) \otimes \boldsymbol{\nabla} \sigma^{2}\right) \\
& +a_{p}\left(\mathbf{G}-\mathbf{G}^{h},\left(\zeta-\zeta^{h}\right) \sigma^{2}\right) \\
& =\left(\zeta-\zeta^{h}, \operatorname{Cof}(\operatorname{Id}+\nabla \mathbf{u}):\left(\mathbf{G}-\mathbf{G}^{h}\right) \otimes \boldsymbol{\nabla} \sigma^{2}\right) \\
& +a_{p}\left(\mathbf{G}-\mathbf{G}^{h},\left(\zeta-\mathcal{Q}_{h} \zeta\right) \sigma^{2}\right)+a_{p}\left(\mathbf{G}-\mathbf{G}^{h}, \xi\right) .
\end{aligned}
$$

The last term on the right-hand side is rewritten by using the weak equations of $\mathbf{G}$ and $\mathbf{G}^{h}$ :

$$
\begin{aligned}
a_{p}\left(\mathbf{G}-\mathbf{G}^{h}, \xi\right) & =a_{p}\left(\mathbf{G}-\mathbf{G}^{h}, \xi-\mathcal{Q}_{h} \xi\right)+a_{p}\left(\mathbf{G}-\mathbf{G}^{h}, \mathcal{Q}_{h} \xi\right) \\
& =a_{p}\left(\mathbf{G}-\mathbf{G}^{h}, \xi-\mathcal{Q}_{h} \xi\right)+\lambda^{-1}\left(\mathcal{Q}_{h} \xi, \zeta-\zeta^{h}\right) \\
& =a_{p}\left(\mathbf{G}-\mathbf{G}^{h}, \xi-\mathcal{Q}_{h} \xi\right)+\lambda^{-1}\left(\mathcal{Q}_{h} \xi, \mathcal{Q}_{h} \zeta-\zeta^{h}\right) .
\end{aligned}
$$

The last two equations yield

$$
\begin{aligned}
a_{p}\left(\boldsymbol{\psi}, \zeta-\zeta^{h}\right)- & \lambda^{-1}\left(\mathcal{Q}_{h} \zeta-\zeta^{h}, \xi\right) \\
= & a_{p}\left(\mathbf{G}-\mathbf{G}^{h},\left(\zeta-\mathcal{Q}_{h} \zeta\right) \sigma^{2}\right)+a_{p}\left(\mathbf{G}-\mathbf{G}^{h}, \xi-\mathcal{Q}_{h} \xi\right) \\
& +\left(\zeta-\zeta^{h}, \operatorname{Cof}(\mathrm{Id}+\boldsymbol{\nabla} \mathbf{u}):\left(\mathbf{G}-\mathbf{G}^{h}\right) \otimes \nabla \sigma^{2}\right) .
\end{aligned}
$$

The first two terms on the right-hand side of A.15 are bounded by

$$
C\left\|\nabla\left(\mathbf{G}-\mathbf{G}^{h}\right)\right\|_{\sigma^{2}}\left(\left\|\zeta-\mathcal{Q}_{h} \zeta\right\|_{\sigma^{2}}+\left\|\xi-\mathcal{Q}_{h} \xi\right\|_{\sigma^{-2}}\right) .
$$

The superapproximation property (4.15) together with (A.11) yields

$$
\left\|\xi-\mathcal{Q}_{h} \xi\right\|_{\sigma^{-2}} \leq C h\left\|\mathcal{Q}_{h} \zeta-\zeta^{h}\right\|_{0} \leq C h^{2}\left(\left\|\mathcal{D}^{2} \mathbf{G}\right\|_{0}+\|\nabla \zeta\|_{0}\right) .
$$

Inserting this into (A.16) and recalling the approximation property (4.14), we obtain a bound of (A.16) that is rewritten by using Young's inequality:

$$
\begin{aligned}
& C\left\|\boldsymbol{\nabla}\left(\mathbf{G}-\mathbf{G}^{h}\right)\right\|_{\sigma^{2}}\left(h\|\boldsymbol{\nabla} \zeta\|_{\sigma^{2}}+h^{2}\left(\left\|\mathcal{D}^{2} \mathbf{G}\right\|_{0}+\|\boldsymbol{\nabla} \zeta\|_{0}\right)\right) \\
& \leq \epsilon\left\|\boldsymbol{\nabla}\left(\mathbf{G}-\mathbf{G}^{h}\right)\right\|_{\sigma^{2}}^{2}+\frac{C}{4 \epsilon}\left(h^{2}\|\boldsymbol{\nabla} \zeta\|_{\sigma^{2}}^{2}+h^{4}\left\|\mathcal{D}^{2} \mathbf{G}\right\|_{0}^{2}+h^{4}\|\boldsymbol{\nabla} \zeta\|_{0}^{2}\right) .
\end{aligned}
$$

Here $\epsilon$ can be any positive number. Notice that $\operatorname{Cof}(\operatorname{Id}+\boldsymbol{\nabla u}):\left(\mathbf{G}-\mathbf{G}^{h}\right) \otimes \boldsymbol{\nabla} \sigma^{2} \in \mathbf{X}$. Using Young's inequality once again, we estimate the fourth term of A.15):

$$
\begin{aligned}
\left\|\zeta-\zeta^{h}\right\|_{-1} & \left\|\boldsymbol{\nabla}\left(\operatorname{Cof}(\mathrm{Id}+\boldsymbol{\nabla} \mathbf{u}):\left(\mathbf{G}-\mathbf{G}^{h}\right) \otimes \boldsymbol{\nabla} \sigma^{2}\right)\right\|_{0} \\
& \leq C\left\|\zeta-\zeta^{h}\right\|_{-1}\left(\left\|\mathbf{G}-\mathbf{G}^{h}\right\|_{0}+\left\|\mathbf{G}-\mathbf{G}^{h}\right\|_{\sigma^{2}}+\left\|\boldsymbol{\nabla}\left(\mathbf{G}-\mathbf{G}^{h}\right)\right\|_{\sigma^{2}}\right) \\
& \leq \epsilon\left\|\boldsymbol{\nabla}\left(\mathbf{G}-\mathbf{G}^{h}\right)\right\|_{\sigma^{2}}^{2}+\frac{C}{4 \epsilon} h^{4}\left(\left\|\mathcal{D}^{2} \mathbf{G}\right\|_{0}+\|\boldsymbol{\nabla} \zeta\|_{0}\right)^{2} .
\end{aligned}
$$

This and (A.17) yield the required estimate (A.14).

We start the discussion of weighted norms with a direct corollary of [15, Lemma 3.3]. 
Lemma A.8. Let $p \in \mathcal{C}^{1}(\bar{\Omega})$. Then for any $\rho \in W^{1, \infty}(\Omega)$ with $\rho \geq 0$, we have

$$
a_{\mathbf{u}}\left(\mathbf{v}, \mathbf{v} \rho^{2}\right)+C \int_{\Omega} \mathbf{v}^{2}\left(\rho^{2}+|\nabla \rho|^{2}\right) d \mathbf{x} \geq C \int_{\Omega}|\nabla \mathbf{v}|^{2} \rho^{2} d \mathbf{x} .
$$

Lemma A.9. If $h$ is sufficiently small, then

$$
\left\|\boldsymbol{\nabla}\left(\mathbf{G}-\mathbf{G}^{h}\right)\right\|_{\sigma^{2}}+\lambda^{-1 / 2}\left\|\zeta-\zeta^{h}\right\|_{\sigma^{2}} \leq C h^{2}\left(\left\|\mathcal{D}^{2} \mathbf{G}\right\|_{0}+\|\boldsymbol{\nabla} \zeta\|_{0}\right)
$$

$$
+C h\left(\left\|\mathcal{D}^{2} \mathbf{G}\right\|_{\sigma^{2}}+\|\nabla \zeta\|_{\sigma^{2}}\right)+C\left(h\left\|\zeta-\zeta^{h}\right\|_{\sigma^{2}}+\left\|\mathbf{G}-\mathbf{G}^{h}\right\|_{\sigma^{2}}\right) .
$$

Proof. Setting again $\boldsymbol{\psi}:=\left(\mathbf{G}-\mathbf{G}^{h}\right) \sigma^{2}$ and $\xi:=\left(\mathcal{Q}_{h} \zeta-\zeta^{h}\right) \sigma^{2}$, we have

$$
\begin{aligned}
& a_{\mathbf{u}}\left(\mathbf{G}-\mathbf{G}^{h},\left(\mathbf{G}-\mathbf{G}^{h}\right) \sigma^{2}\right)+\lambda^{-1}\left(\mathcal{Q}_{h} \zeta-\zeta^{h},\left(\mathcal{Q}_{h} \zeta-\zeta^{h}\right) \sigma^{2}\right) \\
& =a_{\mathbf{u}}\left(\mathbf{G}-\mathbf{G}^{h}, \boldsymbol{\psi}-\boldsymbol{\Pi}_{h} \boldsymbol{\psi}\right)-a_{p}\left(\boldsymbol{\Pi}_{h} \boldsymbol{\psi}, \zeta-\zeta^{h}\right)+\lambda^{-1}\left(\mathcal{Q}_{h} \zeta-\zeta^{h}, \xi\right) \\
& =a_{\mathbf{u}}\left(\mathbf{G}-\mathbf{G}^{h}, \boldsymbol{\psi}-\boldsymbol{\Pi}_{h} \boldsymbol{\psi}\right)+a_{p}\left(\boldsymbol{\psi}-\mathbf{\Pi}_{h} \boldsymbol{\psi}, \zeta-\zeta^{h}\right) \\
& +\lambda^{-1}\left(\mathcal{Q}_{h} \zeta-\zeta^{h}, \xi\right)-a_{p}\left(\boldsymbol{\psi}, \zeta-\zeta^{h}\right) .
\end{aligned}
$$

By recalling (4.3), we decompose the second term on the right-hand side of the above identity:

$$
\begin{aligned}
\left|a_{p}\left(\boldsymbol{\psi}-\boldsymbol{\Pi}_{h} \boldsymbol{\psi}, \zeta-\zeta^{h}\right)\right|= & \mid\left(\zeta-\zeta^{h}, \operatorname{div}\left(\left(\operatorname{adj}-\operatorname{adj}_{h}\right)(\operatorname{Id}+\boldsymbol{\nabla} \mathbf{u})\left(\boldsymbol{\psi}-\boldsymbol{\Pi}_{h} \boldsymbol{\psi}\right)\right)\right) \\
& +\left(\zeta-\mathcal{Q}_{h} \zeta, \operatorname{div}\left(\operatorname{adj}_{h}(\operatorname{Id}+\boldsymbol{\nabla} \mathbf{u})\left(\boldsymbol{\psi}-\boldsymbol{\Pi}_{h} \boldsymbol{\psi}\right)\right)\right) \mid \\
\leq & C\left(h\left\|\zeta-\zeta^{h}\right\|_{\sigma^{2}}+\left\|\zeta-\mathcal{Q}_{h} \zeta\right\|_{\sigma^{2}}\right)\left\|\boldsymbol{\nabla}\left(\boldsymbol{\psi}-\boldsymbol{\Pi}_{h} \boldsymbol{\psi}\right)\right\|_{\sigma^{-2}}
\end{aligned}
$$

From Lemma A.7 and A.21 it follows that for any $\epsilon>0$,

$$
\begin{aligned}
& a_{\mathbf{u}}\left(\mathbf{G}-\mathbf{G}^{h},\left(\mathbf{G}-\mathbf{G}^{h}\right) \sigma^{2}\right)+\lambda^{-1}\|\xi\|_{\sigma^{2}}^{2} \\
& \leq C\left(\left\|\boldsymbol{\nabla}\left(\mathbf{G}-\mathbf{G}^{h}\right)\right\|_{\sigma^{2}}+h\left\|\zeta-\zeta^{h}\right\|_{\sigma^{2}}+h\|\nabla \zeta\|_{\sigma^{2}}\right)\left\|\boldsymbol{\nabla}\left(\boldsymbol{\psi}-\boldsymbol{\Pi}_{h} \boldsymbol{\psi}\right)\right\|_{\sigma^{-2}} \\
& +\epsilon\left\|\boldsymbol{\nabla}\left(\mathbf{G}-\mathbf{G}^{h}\right)\right\|_{\sigma^{2}}^{2}+C(\epsilon) h^{4}\left(\left\|\mathcal{D}^{2} \mathbf{G}\right\|_{0}+\|\boldsymbol{\nabla} \zeta\|_{0}\right)^{2}+C(\epsilon) h^{2}\|\boldsymbol{\nabla} \zeta\|_{\sigma^{2}}
\end{aligned}
$$

The weighted Gårding inequality (A.18) yields

$$
\begin{aligned}
C\left\|\nabla\left(\mathbf{G}-\mathbf{G}^{h}\right)\right\|_{\sigma^{2}}^{2} & \leq a_{\mathbf{u}}\left(\mathbf{G}-\mathbf{G}^{h},\left(\mathbf{G}-\mathbf{G}^{h}\right) \sigma^{2}\right) \\
& +C\left(\left\|\mathbf{G}-\mathbf{G}^{h}\right\|_{\sigma^{2}}^{2}+\left\|\mathbf{G}-\mathbf{G}^{h}\right\|_{0}^{2}\right) .
\end{aligned}
$$

We substitute (A.23) into (A.22). Now Lemma A.6 as well as Lemma A.5 result in

$$
\begin{aligned}
& \left\|\boldsymbol{\nabla}\left(\mathbf{G}-\mathbf{G}^{h}\right)\right\|_{\sigma^{2}}^{2}+\lambda^{-1}\left\|\zeta-\zeta^{h}\right\|_{\sigma^{2}}^{2} \leq\left(\frac{C}{K}+\epsilon\right)\left\|\boldsymbol{\nabla}\left(\mathbf{G}-\mathbf{G}^{h}\right)\right\|_{\sigma^{2}}^{2} \\
& +C(\epsilon) h^{4}\left(\left\|\mathcal{D}^{2} \mathbf{G}\right\|_{0}+\|\boldsymbol{\nabla} \zeta\|_{0}\right)^{2}+\left(C_{K}+C(\epsilon)\right) h^{2}\left(\left\|\mathcal{D}^{2} \mathbf{G}\right\|_{\sigma^{2}}+\|\boldsymbol{\nabla} \zeta\|_{\sigma^{2}}\right)^{2} \\
& +C h^{2}\left\|\zeta-\zeta^{h}\right\|_{\sigma^{2}}^{2}+C\left\|\mathbf{G}-\mathbf{G}^{h}\right\|_{\sigma^{2}}^{2} .
\end{aligned}
$$

Let $K$ be big enough, e.g., $K:=4 C$, and choose $\epsilon:=1 / 4$. Now the first term on the right-hand side of A.24 can be absorbed by spending a factor of 2 . This completes the proof of A.19.

Next, in order to estimate the last two terms on the right-hand side of (A.19), we apply a duality argument. First, for any $\mathbf{w}, \mathbf{v} \in \mathbf{X}$ and $r \in M$, we define

$$
\begin{aligned}
{[\mathbf{w}, \mathbf{v}]_{\mathbf{u}} } & :=a_{\mathbf{u}}\left(\mathbf{w}, \sigma^{2} \mathbf{v}\right)-a_{\mathbf{u}}\left(\sigma^{2} \mathbf{w}, \mathbf{v}\right), \\
{[r, \mathbf{v}]_{p} } & :=a_{p}\left(r, \sigma^{2} \mathbf{v}\right)-a_{p}\left(\sigma^{2} r, \mathbf{v}\right)
\end{aligned}
$$


A direct manipulation yields the following two identities:

$$
\begin{aligned}
{[\mathbf{w}, \mathbf{v}]_{\mathbf{u}}: } & =\left(\boldsymbol{\nabla} \mathbf{w}, \mathbf{v} \otimes \boldsymbol{\nabla} \sigma^{2}\right)-\left(\mathbf{w} \otimes \boldsymbol{\nabla} \sigma^{2}, \mathbf{v}\right) \\
& -\left(\boldsymbol{\nabla} p,\left(\boldsymbol{\nabla} \sigma^{2}\right)^{\perp} \otimes \mathbf{w}^{\perp} \mathbf{v}\right), \\
{[r, \mathbf{v}]_{p}: } & =-\left(r, \operatorname{Cof}(\operatorname{Id}+\boldsymbol{\nabla} \mathbf{u}): \boldsymbol{\nabla} \sigma^{2} \otimes \mathbf{v}\right) .
\end{aligned}
$$

Lemma A.10.

$$
\begin{aligned}
\left\|\zeta-\zeta^{h}\right\|_{\sigma^{2}} & \leq C\left(\left\|\nabla\left(\mathbf{G}-\mathbf{G}^{h}\right)\right\|_{\sigma^{2}}+\lambda^{-1 / 2}\left\|\zeta-\zeta^{h}\right\|_{\sigma^{2}}\right) \\
& +C h^{2}\left(\left\|\mathcal{D}^{2} \mathbf{G}\right\|_{0}+\|\boldsymbol{\nabla} \zeta\|_{0}\right)+C h\|\boldsymbol{\nabla} \zeta\|_{\sigma^{2}}
\end{aligned}
$$

Proof. Consider the following auxiliary problem: Find $(\mathbf{w}, r) \in \mathbf{X} \times M$ such that

$$
\mathcal{A}(\mathbf{w}, r ; \mathbf{v}, q)=\left(\zeta-\zeta^{h}, q\right), \quad \forall(\mathbf{v}, q) \in \mathbf{X} \times M .
$$

Lemma 3.2 and Lemma 3.4 provide the following a priori estimates:

$$
\begin{aligned}
\|\mathbf{w}\|_{0}+\|r\|_{-1} & \leq C\left\|\zeta-\zeta^{h}\right\|_{-1}, \\
\|\nabla \mathbf{w}\|_{0}+\|r\|_{0} & \leq C\left\|\zeta-\zeta^{h}\right\|_{0}, \\
\|\nabla \mathbf{w}\|_{\sigma^{2}}+\|r\|_{\sigma^{2}} & \leq C\left(\left\|\zeta-\zeta^{h}\right\|_{-1}+h\left\|\zeta-\zeta^{h}\right\|_{0}+\left\|\zeta-\zeta^{h}\right\|_{\sigma^{2}}\right) .
\end{aligned}
$$

Set $q:=\left(\zeta-\zeta^{h}\right) \sigma^{2}$ and $\mathbf{v}:=\left(\mathbf{G}-\mathbf{G}^{h}\right) \sigma^{2}$ in (A.27).

$$
\left\|\zeta-\zeta^{h}\right\|_{\sigma^{2}}^{2}=\mathcal{A}\left(\mathbf{w}, r ;\left(\mathbf{G}-\mathbf{G}^{h}\right) \sigma^{2},\left(\zeta-\zeta^{h}\right) \sigma^{2}\right)=\mathcal{A}\left(\sigma^{2} \mathbf{w}, \sigma^{2} r ; \mathbf{G}-\mathbf{G}^{h}, \zeta-\zeta^{h}\right)
$$

$$
+\left[\mathbf{w}, \mathbf{G}-\mathbf{G}^{h}\right]_{\mathbf{u}}+\left[r, \mathbf{G}-\mathbf{G}^{h}\right]_{p}+\left[\zeta-\zeta^{h}, \mathbf{w}\right]_{p} .
$$

We decompose the first term on the right-hand side of A.29) as

$$
\begin{aligned}
\mathcal{A}\left(\sigma^{2} \mathbf{w}, \sigma^{2} r ; \mathbf{G}-\mathbf{G}^{h}, \zeta-\zeta^{h}\right) & \\
& =\mathcal{A}\left(\sigma^{2} \mathbf{w}-\mathbf{\Pi}_{h}\left(\sigma^{2} \mathbf{w}\right), \sigma^{2} r-\mathcal{Q}_{h}\left(\sigma^{2} r\right) ; \mathbf{G}-\mathbf{G}^{h}, \zeta-\zeta^{h}\right) .
\end{aligned}
$$

Similarly to as in (A.21), the expression $a_{p}\left(\sigma^{2} \mathbf{w}-\mathbf{\Pi}_{h}\left(\sigma^{2} \mathbf{w}\right), \zeta-\zeta^{h}\right)$ can be bounded by

$$
C\left(h\left\|\zeta-\zeta^{h}\right\|_{\sigma^{2}}+\left\|\zeta-\mathcal{Q}_{h} \zeta\right\|_{\sigma^{2}}\right)\left\|\nabla\left(\sigma^{2} \mathbf{w}-\boldsymbol{\Pi}_{h}\left(\sigma^{2} \mathbf{w}\right)\right)\right\|_{\sigma^{-2}} .
$$

From (A.25) and (A.29) it follows that

$$
\begin{aligned}
\left\|\zeta-\zeta^{h}\right\|_{\sigma^{2}}^{2} & \leq C\left(\left\|\boldsymbol{\nabla}\left(\mathbf{G}-\mathbf{G}^{h}\right)\right\|_{\sigma^{2}}+\lambda^{-1 / 2}\left\|\zeta-\zeta^{h}\right\|_{\sigma^{2}}\right) \\
& \times\left(\left\|\boldsymbol{\nabla}\left(\sigma^{2} \mathbf{w}-\mathbf{\Pi}_{h}\left(\sigma^{2} \mathbf{w}\right)\right)\right\|_{\sigma^{-2}}+\left\|\sigma^{2} r-\mathcal{Q}_{h}\left(\sigma^{2} r\right)\right\|_{\sigma^{-2}}\right) \\
& +C\left(h\left\|\zeta-\zeta^{h}\right\|_{\sigma^{2}}+\left\|\zeta-\mathcal{Q}_{h} \zeta\right\|_{\sigma^{2}}\right)\left\|\boldsymbol{\nabla}\left(\sigma^{2} \mathbf{w}-\mathbf{\Pi}_{h}\left(\sigma^{2} \mathbf{w}\right)\right)\right\|_{\sigma^{-2}} \\
& +C\left\|\boldsymbol{\nabla}\left(\mathbf{G}-\mathbf{G}^{h}\right)\right\|_{\sigma^{2}}\|\mathbf{w}\|_{0}+C\left\|\mathbf{G}-\mathbf{G}^{h}\right\|_{0}\left(\|\boldsymbol{\nabla} \mathbf{w}\|_{\sigma^{2}}+\|r\|_{\sigma^{2}}\right)
\end{aligned}
$$

A simple calculation yields

$\left\|\boldsymbol{\nabla}\left(\sigma^{2} \mathbf{w}-\Pi_{h}\left(\sigma^{2} \mathbf{w}\right)\right)\right\|_{\sigma^{-2}}+\left\|\sigma^{2} r-\mathcal{Q}_{h}\left(\sigma^{2} r\right)\right\|_{\sigma^{-2}} \leq C\left(\|\mathbf{w}\|_{0}+\|\nabla \mathbf{w}\|_{\sigma^{2}}+\|r\|_{\sigma^{2}}\right)$.

Inserting this into the right-hand side of (A.30) and using Lemma A.5 as well as (A.28), we obtain A.26).

To facilitate the subsequent analysis, we introduce another norm:

$$
\left\|\mathbf{G}-\mathbf{G}^{h}, \zeta-\zeta^{h}\right\|:=\left\|\nabla\left(\mathbf{G}-\mathbf{G}^{h}\right)\right\|_{\sigma^{2}}+\left\|\zeta-\zeta^{h}\right\|_{\sigma^{2}} .
$$

Lemma A.11. For arbitrary $\epsilon>0$, there exists a constant $C(\epsilon)$ which is independent of $h$ such that for sufficiently small $h$, it holds that

$$
\left\|\mathbf{G}-\mathbf{G}^{h}\right\|_{\sigma^{2}} \leq C(h+\epsilon)\left\|\mathbf{G}-\mathbf{G}^{h}, \zeta-\zeta^{h}\right\| \mid C(\epsilon)\left\|\mathbf{G}-\mathbf{G}^{h}\right\|_{0} .
$$


Proof. We consider the following auxiliary problem: Find $(\mathbf{w}, r) \in \mathbf{X} \times M$ such that

$$
\mathcal{A}(\mathbf{w}, r ; \mathbf{v}, q)=\left\langle\mathbf{G}-\mathbf{G}^{h}, \mathbf{v}\right\rangle, \quad \forall(\mathbf{v}, q) \in \mathbf{X} \times M .
$$

From Theorem 3.1 we have the regularity estimates

$$
\begin{aligned}
\|\mathbf{w}\|_{2}+\|r\|_{1} & \leq C\left\|\mathbf{G}-\mathbf{G}^{h}\right\|_{0}, \\
\left\|\mathcal{D}^{2} \mathbf{w}\right\|_{\sigma^{2}}+\|\nabla r\|_{\sigma^{2}} & \leq C\left(\left\|\mathbf{G}-\mathbf{G}^{h}\right\|_{0}+\left\|\mathbf{G}-\mathbf{G}^{h}\right\|_{\sigma^{2}}\right) .
\end{aligned}
$$

Inserting $\left(\mathbf{G}-\mathbf{G}^{h}\right) \sigma^{2}$ into the right-hand side of (A.32) and proceeding along the same lines as in Lemma A.10, we get

$$
\begin{aligned}
\left\|\mathbf{G}-\mathbf{G}^{h}\right\|_{\sigma^{2}}^{2} & =\mathcal{A}\left(\sigma^{2} \mathbf{w}-\mathbf{\Pi}_{h}\left(\sigma^{2} \mathbf{w}\right), \sigma^{2} s-\mathcal{Q}_{h}\left(\sigma^{2} s\right) ; \mathbf{G}-\mathbf{G}^{h}, \zeta-\zeta^{h}\right) \\
& +\left[\mathbf{w}, \mathbf{G}-\mathbf{G}^{h}\right]_{\mathbf{u}}+\left[s, \mathbf{G}-\mathbf{G}^{h}\right]_{p}+\left[\zeta-\zeta^{h}, \mathbf{w}\right]_{p} .
\end{aligned}
$$

From (3.10) and Hölder's inequality it follows that

$$
\|\mathbf{w}\|_{\sigma^{-2}} \leq\left(\int_{\Omega}|\mathbf{w}(\mathbf{x})|^{2 s} d \mathbf{x}\right)^{1 / 2 s}\left(\int_{\Omega} \sigma(\mathbf{x})^{-2 s /(s-1)} d \mathbf{x}\right)^{(s-1) / 2 s} \leq C s^{1 / 2} \theta^{-1 / s}\|\mathbf{w}\|_{1},
$$

where we have used $\|\mathbf{w}\|_{L^{2 s}} \leq C s^{1 / 2}\|\mathbf{w}\|_{1}$. Setting $s=|\ln \theta|$ in the above inequality, recalling that $K$ has been fixed in A.24), and using Poincaré's inequality, we obtain

$$
\|\mathbf{w}\|_{\sigma^{-2}} \leq C|\ln h|^{1 / 2}\|\nabla \mathbf{w}\|_{0} .
$$

Applying the weighted approximation property 4.5 with $j=2$ and $k=1$ and recalling (A.36), we have the approximation estimate

$$
\begin{aligned}
\left\|\boldsymbol{\nabla}\left(\sigma^{2} \mathbf{w}-\boldsymbol{\Pi}_{h}\left(\sigma^{2} \mathbf{w}\right)\right)\right\|_{\sigma^{-2}} & \leq C h\left\|\mathcal{D}^{2}\left(\sigma^{2} \mathbf{w}\right)\right\|_{\sigma^{-2}} \\
& \leq C h\left(|\ln h|^{1 / 2}\|\boldsymbol{\nabla} \mathbf{w}\|_{0}+\left\|\mathcal{D}^{2} \mathbf{w}\right\|_{\sigma^{2}}\right),
\end{aligned}
$$

and invoking the weighted approximation property (4.14), we obtain

$$
\left\|\sigma^{2} r-\mathcal{Q}_{h}\left(\sigma^{2} r\right)\right\|_{\sigma^{-2}} \leq C h\left(\|r\|_{0}+\|\nabla r\|_{\sigma^{2}}\right) .
$$

The last two inequalities provide bounds of the first four terms on the right-hand side of (A.35)

$$
C h\left(\|r\|_{0}+\|\boldsymbol{\nabla} r\|_{\sigma^{2}}+|\ln h|^{1 / 2}\|\boldsymbol{\nabla} \mathbf{w}\|_{0}+\left\|\mathcal{D}^{2} \mathbf{w}\right\|_{\sigma^{2}}\right)\left\|\mathbf{G}-\mathbf{G}^{h}, \zeta-\zeta^{h}\right\|,
$$
and the remaining three terms on the right-hand side of (A.35) are bounded by

$$
C\left\|\mathbf{G}-\mathbf{G}^{h}\right\|_{\sigma^{2}}\left(\|\nabla \mathbf{w}\|_{0}+\|r\|_{0}\right)+C\left\|\mathbf{G}-\mathbf{G}^{h}, \zeta-\zeta^{h}\right\|\|\mathbf{w}\|_{0} .
$$

Inserting (A.37) and A.38) into A.35, taking into account (A.33) and A.34), we have

$$
\begin{aligned}
\left\|\mathbf{G}-\mathbf{G}^{h}\right\|_{\sigma^{2}}^{2} & \leq C\left\|\mathbf{G}-\mathbf{G}^{h}, \zeta-\zeta^{h}\right\|\left(h|\ln h|^{1 / 2}\left\|\mathbf{G}-\mathbf{G}^{h}\right\|_{0}+h\left\|\mathbf{G}-\mathbf{G}^{h}\right\|_{\sigma^{2}}\right) \\
& +C\left\|\mathbf{G}-\mathbf{G}^{h}\right\|_{0}^{2}+C\left\|\mathbf{G}-\mathbf{G}^{h}\right\|_{0}\left\|\mathbf{G}-\mathbf{G}^{h}\right\|_{\sigma^{2}} \\
& +C\left\|\zeta-\zeta^{h}\right\|_{\sigma^{2}}\left\|\mathbf{G}-\mathbf{G}^{h}\right\|_{0} .
\end{aligned}
$$

Since $h|\ln h|^{1 / 2} \mid<1$, recalling the definition of $\mid\|\cdot, \cdot\| \|$, we obtain

$$
\begin{aligned}
\left\|\mathbf{G}-\mathbf{G}^{h}\right\|_{\sigma^{2}}^{2} \leq C\left\|\mathbf{G}-\mathbf{G}^{h}, \zeta-\zeta^{h}\right\|\left(\left\|\mathbf{G}-\mathbf{G}^{h}\right\|_{0}+h\left\|\mathbf{G}-\mathbf{G}^{h}\right\|_{\sigma^{2}}\right) \\
+C\left\|\mathbf{G}-\mathbf{G}^{h}\right\|_{0}^{2}+C\left\|\mathbf{G}-\mathbf{G}^{h}\right\|_{0}\left\|\mathbf{G}-\mathbf{G}^{h}\right\|_{\sigma^{2}} .
\end{aligned}
$$

Young's inequality is applied twice. In particular, the product of the first two factors on the right-hand side is dealt with by using the $\epsilon$-technique to obtain (A.31). 
Now we are in a position to prove Lemma 5.4 and Theorem 5.5 .

Proof of Lemma 5.4 By Theorem 3.3 we only need to estimate $\left\|\boldsymbol{\delta}_{1}\right\|_{\sigma_{1}^{2}}$ and $\left\|\boldsymbol{\delta}_{1}\right\|_{-1}$ to prove (5.14) for $i=1$. The construction of $\boldsymbol{\delta}_{1}$ implies that $\left\|\boldsymbol{\delta}_{1}\right\|_{\sigma_{1}^{2}}=\mathcal{O}(1)$. Next, set $\boldsymbol{\pi}_{h} \mathbf{v}:=\left(I_{h} v_{1}, I_{h} v_{2}\right)$, where $I_{h}$ is the Clément interpolation operator 13. Let $\mathcal{B}$ be the unit ball of $\mathbf{H}_{0}^{1}(\Omega)$. By the Sobolev imbedding inequality in $2 \mathrm{D}$, we have

$$
\|v\|_{L^{s}} \leq C s^{1 / 2}\|v\|_{1}, \quad \forall v \in H^{1}(\Omega), \quad 2<s<\infty .
$$

The inverse inequality for finite element spaces [11, p. 140] together with (A.39) yields

$$
\|v\|_{L^{\infty}} \leq C h^{-2 / s}\|v\|_{L^{s}} \leq C h^{-2 / s} s^{1 / 2}\|v\|_{1} .
$$

Setting $s=|\ln h|$ in the above inequality, we get

$$
\|v\|_{L^{\infty}} \leq C|\ln h|^{1 / 2}\|v\|_{1} .
$$

We conclude that

$$
\begin{aligned}
\left\|\boldsymbol{\delta}_{1}\right\|_{-1} & =\sup _{\mathbf{v} \in \mathcal{B}}\left(\boldsymbol{\delta}_{1}, \mathbf{v}\right) \leq \sup _{\mathbf{v} \in \mathcal{B}}\left(\boldsymbol{\delta}_{1}, \mathbf{v}-\boldsymbol{\pi}_{h} \mathbf{v}\right)+\sup _{\mathbf{v} \in \mathcal{B}}\left(\boldsymbol{\delta}_{1}, \boldsymbol{\pi}_{h} \mathbf{v}\right) \\
& \leq \sup _{\mathbf{v} \in \mathcal{B}}\left(C+\left\|\boldsymbol{\pi}_{h} \mathbf{v}\right\|_{L^{\infty}}\right) \leq C+C|\ln h|^{1 / 2} .
\end{aligned}
$$

This proves (5.14). Proceeding along the same lines, we get (5.14) for $i=2,3$.

To prove (5.15), we first consider the case $i=2$. By Lemma 3.4 we have

$$
\left\|\nabla \mathbf{G}_{2}\right\|_{\sigma_{2}^{2}}+\left\|\zeta_{2}\right\|_{\sigma_{2}^{2}} \leq C\left(\theta\left\|\mathcal{D} \boldsymbol{\delta}_{2}\right\|_{-1}+\sum_{j=1}^{2}\left\|\mu_{j} \mathcal{D} \boldsymbol{\delta}_{2}\right\|_{-1}+\left\|\mathcal{D} \boldsymbol{\delta}_{2}\right\|_{-2}\right) .
$$

An integration by parts yields

$$
\left\|\mu_{j} \mathcal{D} \boldsymbol{\delta}_{2}\right\|_{-1} \leq\left\|\boldsymbol{\delta}_{2}\right\|_{-1}+\left\|\mu_{j} \boldsymbol{\delta}_{2}\right\|_{0}
$$

A combination of the above two inequalities gives

$$
\left\|\nabla \mathbf{G}_{2}\right\|_{\sigma_{2}^{2}}+\left\|\zeta_{2}\right\|_{\sigma_{2}^{2}} \leq C\left(\theta\left\|\boldsymbol{\delta}_{2}\right\|_{0}+\sum_{j=1}^{2}\left\|\mu_{j} \boldsymbol{\delta}_{2}\right\|_{0}+\left\|\boldsymbol{\delta}_{2}\right\|_{-1}\right) \leq C|\ln h|^{1 / 2}
$$

In the same way, we get (5.15) for $i=1,3$.

Proof of Theorem 5.5. As a first step, we insert (A.26) into (A.19), and for sufficiently small $h$, we have

$\left\|\boldsymbol{\nabla}\left(\mathbf{G}-\mathbf{G}^{h}\right)\right\|_{\sigma^{2}}+\lambda^{-1 / 2}\left\|\zeta-\zeta^{h}\right\|_{\sigma^{2}} \leq C h^{2}\left(\left\|\mathcal{D}^{2} \mathbf{G}\right\|_{0}+\|\nabla \zeta\|_{0}\right)$

$$
+C h\left(\left\|\mathcal{D}^{2} \mathbf{G}\right\|_{\sigma^{2}}+\|\boldsymbol{\nabla} \zeta\|_{\sigma^{2}}\right)+C\left\|\mathbf{G}-\mathbf{G}^{h}\right\|_{\sigma^{2}} .
$$

Secondly, inserting (A.40) into (A.26), we have for sufficiently small $h$

$$
\begin{aligned}
\left\|\zeta-\zeta^{h}\right\|_{\sigma^{2}} & \leq C h^{2}\left(\left\|\mathcal{D}^{2} \mathbf{G}\right\|_{0}+\|\nabla \zeta\|_{0}\right)+C h\left(\left\|\mathcal{D}^{2} \mathbf{G}\right\|_{\sigma^{2}}+\|\nabla \zeta\|_{\sigma^{2}}\right) \\
& +C\left\|\mathbf{G}-\mathbf{G}^{h}\right\|_{\sigma^{2}}
\end{aligned}
$$

Inserting (A.31) into (A.40) and (A.41), we get

$$
\begin{aligned}
\left\|\mathbf{G}-\mathbf{G}^{h}, \zeta-\zeta^{h}\right\| & \leq C(h+\epsilon)\left\|\mathbf{G}-\mathbf{G}^{h}, \zeta-\zeta^{h}\right\| \\
& +C(\epsilon) h^{2}\left(\left\|\mathcal{D}^{2} \mathbf{G}\right\|_{0}+\|\boldsymbol{\nabla} \zeta\|_{0}\right)+C h\left(\left\|\mathcal{D}^{2} \mathbf{G}\right\|_{\sigma^{2}}+\|\boldsymbol{\nabla} \zeta\|_{\sigma^{2}}\right) .
\end{aligned}
$$


Finally we choose $\epsilon:=1 /(4 C)$. If also $C h<1 / 4$, the first term on the right-hand side can be absorbed by spending a factor of 2. Using Lemma A.4 as well as Lemma 5.4. we complete the proof of Theorem 5.5

\section{ACKNOWLEDGMENTS}

This work was finished when the second author was visiting the Faculty of Mathematics at the Ruhr-University Bochum on a grant from the Alexander von Humboldt Foundation. He would like to thank the Alexander von Humboldt Foundation for their support.

\section{REFERENCES}

1. L. Agmon, L. Douglas, and L. Nirenberg, Estimates near the boundary for solutions of elliptic partial differential equations satisfying general boundary conditions II, Comm. Pure. Appl. Math. 17 (1964), 35-92. MF 28:5252

2. I. Babuška and M. Suri, On locking and robustness in the finite element method, SIAM. J. Numer. Anal. 29 (1992), 1261-1293. MR.94c:65128

3. C. Bernardi and V. Girault, A local regularization operator for triangular and quadrilateral finite elements, SIAM. J. Numer. Anal. 35 (1998), 1893-1916. MR.99g:65107

4. J. M. Boland and R. A. Nicolaides, Stable and semistable low order finite elements for viscous flows, SIAM. J. Numer. Anal. 22 (1985), 474-492. MR.86m:65139

5. D. Braess, Stability of saddle point problems with penalty, RAIRO, M² AN 30 (1996), 731-742. MR99k:65099

6. Enhanced assumed strain elements and locking in membrane problems, Comput. Methods Appl. Mech. Eng. 165 (1998), 155-174. MR2000j:74084

7. _ Finite Elements: Theory, Fast Solvers, and Applications in Solid Mechanics, Cambridge University Press, 2001. MR 2001k:65002

8. D. Braess, C. Carstensen, and B. D. Reddy, Uniform convergence and a posteriori error estimators for the enhanced strain finite element method, Numer. Math. 96 (2004) 461-479.

9. S. C. Brenner and L. R. Scott, The Mathematical Theory of Finite Element Methods, SpringerVerlag, New York, 1994. MF.95f:65001

10. F. Brezzi and M. Fortin, Mixed and Hybrid Finite Element Methods, Springer-Verlag, New York, 1991. MR92d:65187

11. P. G. Ciarlet, Finite Element Methods for Elliptic Problems, North-Holland, Amsterdam, 1978. MR 58:25001

12. _ Mathematical Elasticity. Volume 1: Three-dimensional Elasticity, North-Holland, Amsterdam, 1988. MR89e:73001

13. P. Clément, Approximation by finite element functions using local regularization, RAIRO, Sér. Rouge Anal. Numér. R-2 (1975), 77-84. MR53:4569

14. M. C. Crandall and P. H. Rabinowitz, Bifurcation from simple eigenvalues, J. Funct. Anal. 8 (1971), 321-340. MR 44:5836

15. M. Dobrowolski, A mixed finite element method for approximating incompressible materials, SIAM. J. Numer. Anal. 29 (1992), 365-389. MR93b:65167

16. R. Durán, R. Nochetto, and J. Wang, Sharp maximum norm error estimates for finite element approximations of the Stokes problem in 2-D, Math. Comp. 51 (1988), 491-506. MR89b:65261

17. M. Fortin, An analysis of the convergence of mixed finite element methods, RAIRO, Anal. Numér. 11 (1977), 341-354. MR 57:4473

18. J. Frehse and R. Rannacher, Eine $L^{1}-$ Fehlerabschätzung für diskrete Grundlösungen in der Methode der finiten Elemente, Bonner Math. Schriften. 89 (1976), 92-114. MR57:11104

19. __ Asymptotic $L^{\infty}$ error estimates for linear finite element approximations of quasilinear boundary value problems, SIAM. J. Numer. Anal. 15 (1978), 418-431. MR58:19224

20. P. Grisvard, Elliptic Problems in Nonsmooth Domains, Pitman, Boston, 1985. MR.86m:35044

21. H. Le Dret, Constitutive laws and existence questions in incompressible nonlinear elasticity, J. Elasticity. 15 (1985), 369-387. MR87h:73022

22. - Incompressible limit behaviour of slightly compressible nonlinear elastic materials, RAIRO, M²AN 20 (1986), 315-340. MR87k:73011 
23. P. Le Tallec, Numerical Methods for Nonlinear Three-dimensional Elasticity, in "Handbook of Numerical Analysis", VOL. III. (P. G. Ciarlet and J. L. Lions eds.), 465-662. North-Holland, 1994. MR96b:73093

24. J. L. Lions and E. Magènes, Problèmes aux limites non homogènes et applications (tome 1), S. A. Dunod, Paris, 1968. MR 40:512

25. F. Natterer, Über die punktweise Konvergenz finiter Elemente, Numer. Math. 25 (1975), 67-77. MR 57:14514

26. R. W. Ogden, Large deformation isotropic elasticity: on the correlation of theory and experiment for compressible rubber-like solids, Proc. Roy. Soc. London, A328 (1972), 567-583.

27. R. W. Ogden, Volume changes associated with the deformation of rubber-like solids, J. Mech. Phys. Solids 24 (1976), 323-338.

28. D. Pantuso and K. J. Bathe, On the stability of mixed finite elements in large strain analysis of incompressible solids, Finite Elements in Analysis and Design 28 (1997), 83-104. MR98i:73060

29. J. M. Pouyot, Études numérique de quelques problémes raides en mécanique des milieux faiblement compressible, Calcolo 12 (1975), 275-314. MF 54:9252

30. S. Reese, P. Wriggers, and B.D. Reddy, A new locking-free brick element technique for large deformation problems in elasticity, Computers \& Structures 75 (2000), 291-304. MR2001j:74087

31. W. C. Rheinboldt, Methods for Solving Nonlinear Equations, vol. 14, CBMS Series, SIAM, Philadelpha, 1974. MR51:7278

32. R. Rostamian, Internal constraints in boundary value problems of continuum mechanics, Indiana Math. 27 (1978), 637-656. MR58:14158

33. V. Ruas, Existence and stability of asymmetric finite-element approximations in nonlinear elasticity, SIAM J. Numer. Anal. 26 (1989), 1031-1059. MR90h:65187

34. A. Schatz, An observation concerning Ritz-Galerkin methods with indefinite bilinear forms, Math. Comp. 28 (1974), 959-962. MF 51:9526

35. J. C. Simo and M. S. Rifai, A class of assumed strain methods and the method of incompatible modes, Int. J. Numer. Meths. Engrg. 29 (1990), 1595-1638. MR91d:73062

36. T. Sussman and K. J. Bathe, A finite elment formulation for nonlinear incompressible elastic and noninelastic analysis, Computer \& Structures 26 (1987), 357-409.

37. L. R. Treloar, The Physics of Rubber Elasticity, Oxford University Press, Oxford, 1975.

Faculty of Mathematics, Ruhr-University, 44780 Bochum, Germany

E-mail address: braess@num.ruhr-uni-bochum.de

Institute of Computational Mathematics, Chinese Academy of Sciences, POB 2719, Beijing 100080, Peoples Republic of China

E-mail address: mpb@lsec.cc.ac.cn 